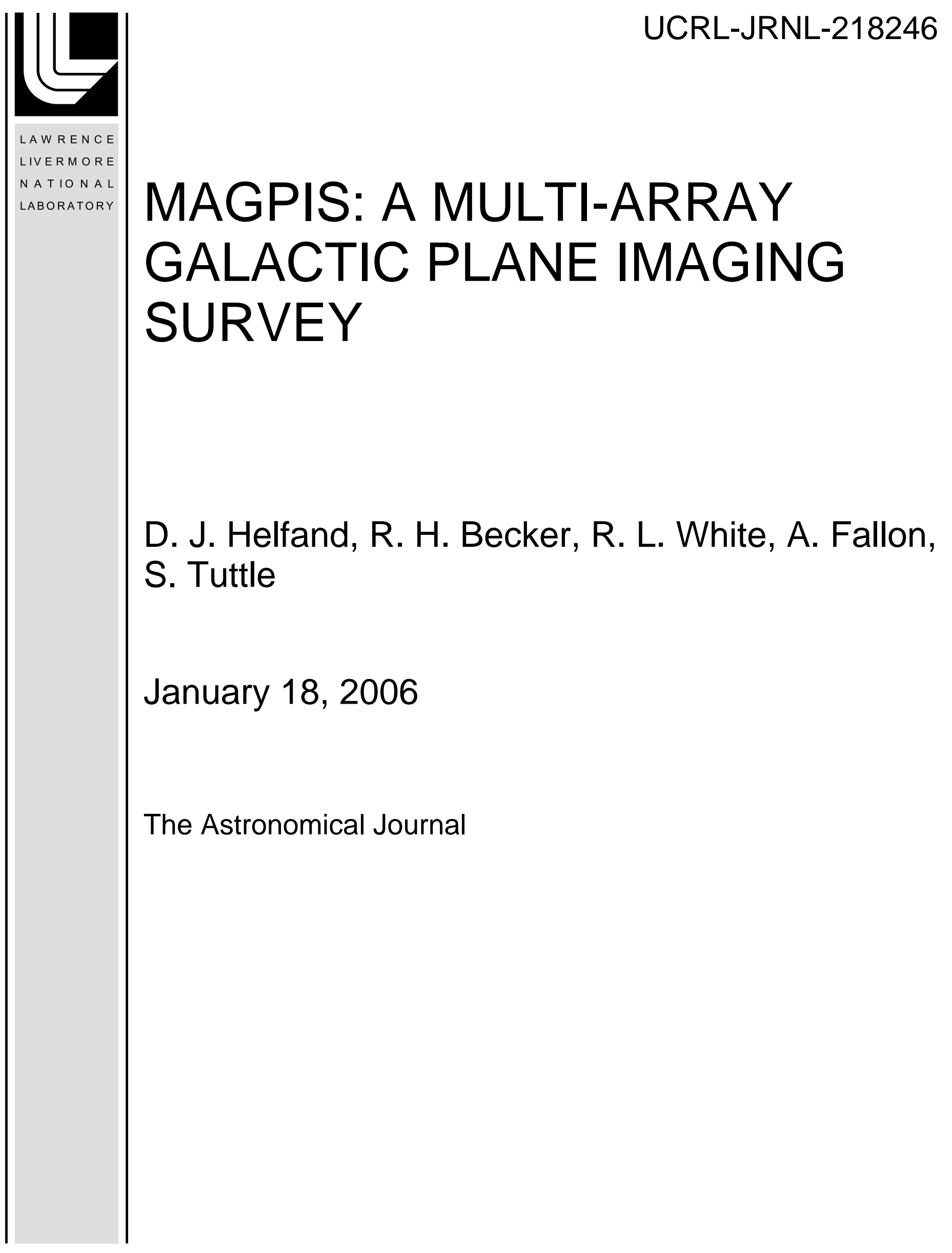


This document was prepared as an account of work sponsored by an agency of the United States Government. Neither the United States Government nor the University of California nor any of their employees, makes any warranty, express or implied, or assumes any legal liability or responsibility for the accuracy, completeness, or usefulness of any information, apparatus, product, or process disclosed, or represents that its use would not infringe privately owned rights. Reference herein to any specific commercial product, process, or service by trade name, trademark, manufacturer, or otherwise, does not necessarily constitute or imply its endorsement, recommendation, or favoring by the United States Government or the University of California. The views and opinions of authors expressed herein do not necessarily state or reflect those of the United States Government or the University of California, and shall not be used for advertising or product endorsement purposes. 
Submitted to the Astronomical Journal (REVISEd 2006.1.24)

Preprint typeset using $\mathrm{LT}_{\mathrm{E}} \mathrm{X}$ style emulateapj v. 11/26/04

\title{
MAGPIS: A MULTI-ARRAY GALACTIC PLANE IMAGING SURVEY
}

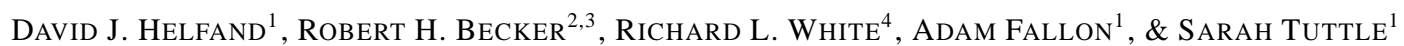 \\ Submitted to the Astronomical Journal (revised 2006.1.24)
}

\begin{abstract}
We present the Multi-Array Galactic Plane Imaging Survey (MAGPIS), which maps portions of the first Galactic quadrant with an angular resolution, sensitivity and dynamic range that surpasses existing radio images of the Milky Way by more than an order of magnitude. The source detection threshold at $20 \mathrm{~cm}$ is in the range $1-2$ mJy over the $85 \%$ of the survey region $\left(5^{\circ}<l<32^{\circ},|b|<0.8\right)$ not covered by bright extended emission; the angular resolution is $\sim 6^{\prime \prime}$. We catalog over 3000 discrete sources (diameters mostly $<30^{\prime \prime}$ ) and present an atlas of $\sim 400$ diffuse emission regions. New and archival data at $90 \mathrm{~cm}$ for the whole survey area are also presented. Comparison of our catalogs and images with the MSX mid-infrared data allow us to provide preliminary discrimination between thermal and non-thermal sources. We identify 49 high-probability supernova remnant candidates, increasing by a factor of seven the number of known remnants with diameters smaller than $5^{\prime}$ in the survey region; several are pulsar wind nebula candidates and/or very small diameter remnants $\left(D<45^{\prime \prime}\right)$. We report the tentative identification of several hundred H II regions based on a comparison with the mid-IR data; they range in size from unresolved ultra-compact sources to large complexes of diffuse emission on scales of half a degree. In several of the latter regions, cospatial nonthermal emission illustrates the interplay between stellar death and birth. We comment briefly on plans for followup observations and our extension of the survey; when complemented by data from ongoing X-ray and mid-IR observations, we expect MAGPIS to provide an important contribution to our understanding of the birth and death of massive stars in the Milky Way.

Subject headings: surveys — catalogs - Galaxy: general — radio continuum: ISM — supernova remnants - HII regions
\end{abstract}

\section{INTRODUCTION}

The Milky Way is a galaxy of stars radiating most of their energy at optical wavelengths. But from stellar birth to stellar death, from the vast reaches of interstellar space to the tiniest of stellar corpses, radio and X-ray observations provide crucial diagnostics in our quest to understand the structure and evolution of our Galaxy and its denizens. These two spectral regimes are particularly crucial for studying massive stars: throughout their lives, stellar Lyman continuum photons produce $\mathrm{H}$ II regions with their associated free-free radio emission, while stellar wind shocks produce X-rays; in death, the remnants of supernovae are the brightest radio and X-ray sources in the Galaxy. Furthermore, the Galaxy is largely transparent in the radio and hard X-ray bands, giving us an unobstructed view through the plane, even at $b=0^{\circ}$. We are in the process of conducting a large-scale survey of the Galactic plane at X-ray wavelengths with XMM, the first results of which have been reported elsewhere (Hands et al. 2004). Here we describe a complementary effort to provide a new, highresolution, high-sensitivity view of centimetric radio emission in the Milky Way's first quadrant.

While significant progress has been made recently in surveying the extragalactic radio sky (e.g., NVSS, SUMSS, and FIRST), the Galactic plane still remains inadequately explored. Even though the NVSS (Condon et al. 1998) covered the plane, it did so in snapshot mode with $u v$ coverage insufficient to achieve high dynamic range (typical values achieved are 30:1). The Canadian Galactic Plane Survey project (En-

\footnotetext{
Electronic address: djh@astro.columbia.edu

${ }^{1}$ Dept. of Astronomy, Columbia University, New York, NY 10027

2 Physics Dept., University of California, Davis, CA 95616

3 IGPP/Lawrence Livermore National Laboratory

${ }^{4}$ Space Telescope Science Institute, Baltimore, MD 21218
}

glish et al. 1998) is covering a large region of the plane in the second quadrant with better dynamic range, but with a resolution of only $65^{\prime \prime}$ and limited sensitivity in the continuum. The VLA Galactic Plane Survey (Taylor et al. 2002) complements this in the longitude range $18^{\circ}<l<67^{\circ}$. The third and fourth Galactic quadrants are being surveyed using Parkes and the Australia Compact Telescope Array in the Southern Galactic Plane Survey (McClure-Griffiths at al. 2001), although the angular resolution is only $\sim 2^{\prime}$ and the $5 \sigma$ detection threshold is $\sim 35 \mathrm{mJy}$ in the continuum.

Fifteen years ago, we used observations originally taken by Dicke et al. (unpublished) in the B-configuration of the Very Large Array $^{5}$ (supplemented by additional $20 \mathrm{~cm}$ and $6 \mathrm{~cm}$ time awarded to us) to produce a catalog of over 4000 compact sources within a degree of the plane in the longitude range $-20^{\circ}<l<120^{\circ}$ (Becker et al. 1990; Zoonematkermani et al. 1990; White, Becker, and Helfand 1991; Helfand et al. 1992). Although the original analysis provided maps that were complete only to $\sim 20 \mathrm{mJy}$ at $20 \mathrm{~cm}$, this remains the highest resolution and most sensitive census of compact sources over a large segment of the Galaxy. Comparison with the IRAS survey led us to identify more than 450 ultracompact $\mathrm{H}$ II regions, over 100 new planetary nebulae (which fill in the gap near $b=0$ caused by extinction in optical searches - Kistiakowsky and Helfand 1995), and, along with $90 \mathrm{~cm}$ maps we obtained covering a small portion of the longitude range, more than a dozen new supernova remnant candidates.

Motivated by the torrent of new, high-resolution midinfrared data from the GLIMPSE Legacy survey with Spitzer (Benjamin et al. 2003) and taking advantage of modern data

\footnotetext{
5 The VLA is a facility of the National Radio Astronomy Observatory which is operated by Associated Universities, Inc. under cooperative agreement with the National Science Foundation.
} 
analysis algorithms developed for our FIRST survey (Becker, White \& Helfand 1995; White et al. 1997), we have recently completed a reanalysis of the snapshot data discussed above (over 3000 individual pointings including some new data designed to fill holes and improve quality in poorly covered regions). This work yielded 6 and $20 \mathrm{~cm}$ catalogs with over 6000 entries and flux density thresholds nearly a factor of two below those of the original analysis (White, Becker \& Helfand 2005). However, the very limited $u v$ coverage afforded by these single-configuration, single-HA, short ( $<3 \mathrm{~min}$ ) observations renders the data problematic for all but the most compact radio sources $\left(<1^{\prime}\right)$ in the plane.

A high-sensitivity, high-resolution, high-dynamic-range map of the radio continuum emission from the Galactic plane can now be constructed with a relatively modest investment of telescope time owing to advances in the VLA receivers over the last decade, the implementation of the highly efficient "survey mode" slewing algorithm, and improvements to the AIPS software package. We have begun to realize this goal by producing a $6^{\prime \prime}$-resolution image of 27 degrees of Galactic longitude in the first quadrant. Our plan over the coming several years is to extend this survey over the entire Spitzer GLIMPSE longitude range in the north, covering $5^{\circ}<l<65^{\circ}$. This Multi-Array Galactic Plane Imaging Survey or MAGPIS (a moniker appropriate for its authors whose careers have been based on collecting random shiny objects gathered by pecking around various corners of celestial sphere in several regions of the electromagnetic spectrum) is designed to provide a definitive archive of the Galactic sky at $20 \mathrm{~cm}$.

In Section 2 we describe the survey parameters and the data acquired to date; in addition, we discuss complementary datasets we have used in our analysis and introduce the MAGPIS website, which offers comprehensive access to all of our data products. Section 3 outlines our analysis strategy, presents the imaging results, and provides a statistcal characterization of the survey sensitivity threshold and dynamic range. We then discuss our detection algorithms for both discrete and diffuse sources, and present the source catalogs as well as an atlas for all extended emission regions (\$4). Section 5 includes a discussion of a preliminary comparison between MAGPIS and the MSX mid-IR data, and previews the prospects for a more complete census of $\mathrm{H}$ II regions in the first quadrant. This is followed by a discussion $(\$ 6)$ of the nonthermal emission regions detected in our survey, including the discovery of several dozen new supernova remnant candidates. We summarize our results in Section 7.

\section{THE MAGPIS SURVEY: DESIGN AND DATA AQUISITION}

As noted above, radio emission is a prominent signature of massive stars; H II regions, pulsars, supernova remnants, and black hole binaries are all the products of $\mathrm{O}$ and early $\mathrm{B}$ stars that have a small scale height. This fact, coupled with constraints on the total observing time available, has led us to restrict our Galactic latitude coverage to $|b|<0$. 8. This is greater than the OB star scale height (Reed 2000) for all distances beyond $3 \mathrm{kpc}$ and covers a region up to $z= \pm 230 \mathrm{pc}$ at the solar circle on the far side of the Galaxy (we adopt $R_{\odot}=$ $8.5 \mathrm{kpc}$ throughout).

\subsection{The $20 \mathrm{~cm}$ data}

Our first tranche of Galactic longitude, $32^{\circ}>l>19^{\circ}$, was chosen to complement our first X-ray data set and to explore the tangent to the Scutum spiral arm. The second segment we
TABLE 1

OBSERVING LOG

\begin{tabular}{ccccc}
\hline \hline & \multicolumn{4}{c}{ VLA Configuration } \\
Description & B & C & D & BnC \\
\hline Phase 1, 20 cm & Mar-Apr 2001 & Aug-Sept 2001 & Aug-Sept 2000 & May 2001 \\
& 31 hrs & 28 hrs & 28 hrs & 1.5 hrs \\
Phase 2, $20 \mathrm{~cm}^{\mathrm{b}}$ & Jan 2004 & Feb-Mar 2004 & Apr 2003 & \\
& $36 \mathrm{hrs}$ & $19 \mathrm{hrs}$ & $18 \mathrm{hrs}$ & \\
Phase 1, $90 \mathrm{~cm}^{\mathrm{c}}$ & & Sept 2001 & & \\
& & $3.5 \mathrm{hrs}$ & \\
& & & \\
\hline
\end{tabular}

${ }^{\mathrm{a}} 20 \mathrm{~cm}$ Phase $1: 19^{\circ}<l<32^{\circ},|b|<0.8$

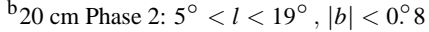

${ }^{\mathrm{c}} 90 \mathrm{~cm}$ Phase 1: $20^{\circ}<l<33^{\circ},|b|<2^{\circ}$

have completed covers the region $19^{\circ}>l>5^{\circ}$; we stopped at $5^{\circ}$ mainly because the central regions have been reasonably well-mapped previously. We intend to continue the survey as time becomes available, first to the GLIMPSE upper longitude limit of $l=65^{\circ}$ and later to both higher and lower longitudes.

Data are collected in the B-, C-, and D-configurations of the VLA operating in pseudo-continuum mode at $20 \mathrm{~cm}$; two $25-$ $\mathrm{MHz}$ bandwidths centered at $1365 \mathrm{MHz}$ and $1435 \mathrm{MHz}$ are broken into seven $3 \mathrm{MHz}$ channels to minimize bandwidth smearing as well as to reduce significantly our sensitivity to interference. This choice entails a loss of a factor of two in bandwidth over the standard continuum mode owing to limitations of the VLA correlator; however, this is not important, since virtually all maps are dynamic-range, rather than sensitivity, limited.

The pointing pattern is displayed in Figure 1. The closepacked hexagonal array provides uniform coverage with a peak-to-minimum variation in sensitivity of $<20 \%$ (after coadding of adjacent images — see Fig. 2). We observe each location four times in each of the three configurations spaced roughly equally in hour angle over a range $\pm 4 \mathrm{hrs}$ to maximize $u v$ coverage; the result is an average of $\sim 12$ minutes per field per configuration, providing a theoretical noise level ( $\sim 0.08 \mathrm{mJy}$ ) far below the dynamic range limit of the maps. In the second round of observations we have saved observing time by using the full 12 minutes per field in the B configuration, but reducing the integration time by a factor of two in the two lower-resolution configurations (while maintaining the observational cadence at multiple hour angles). This reduces our sensitivity by $\sim 20 \%$ in the least-populated map regions, although, again, most of the images are dynamic-range limited and the effect on the final source catalog is minimal.

A total of 165 hours of time has been accumulated to date in the MAGPIS project. Table 1 lists the observing epochs and configurations used to construct the 252 individual images, which cover an area of over $42 \mathrm{deg}^{2}$ with a synthesized beam of 6 ." $2 \times 5$." 4 and a typical $\mathrm{rms}$ of $0.3 \mathrm{mJy}$.

\subsection{The $90 \mathrm{~cm}$ data}

Even with high-quality images, a single frequency is insufficient to identify source classes unambiguously and to disentangle thermal and nonthermal emission in crowded regions. As part of our initial observation program for MAGPIS, we obtained 3.5 hours of $90 \mathrm{~cm}$ pseudo-continuum observations in the $\mathrm{C}$ configuration of the VLA during September of 2001. Eight pointings were used to cover the region $20^{\circ}<l<33^{\circ},|b|<2^{\circ}$. The data were reduced using a $15^{\prime \prime}$ pixel size and have a resolution of $\sim 70^{\prime \prime}$. In addition, we 


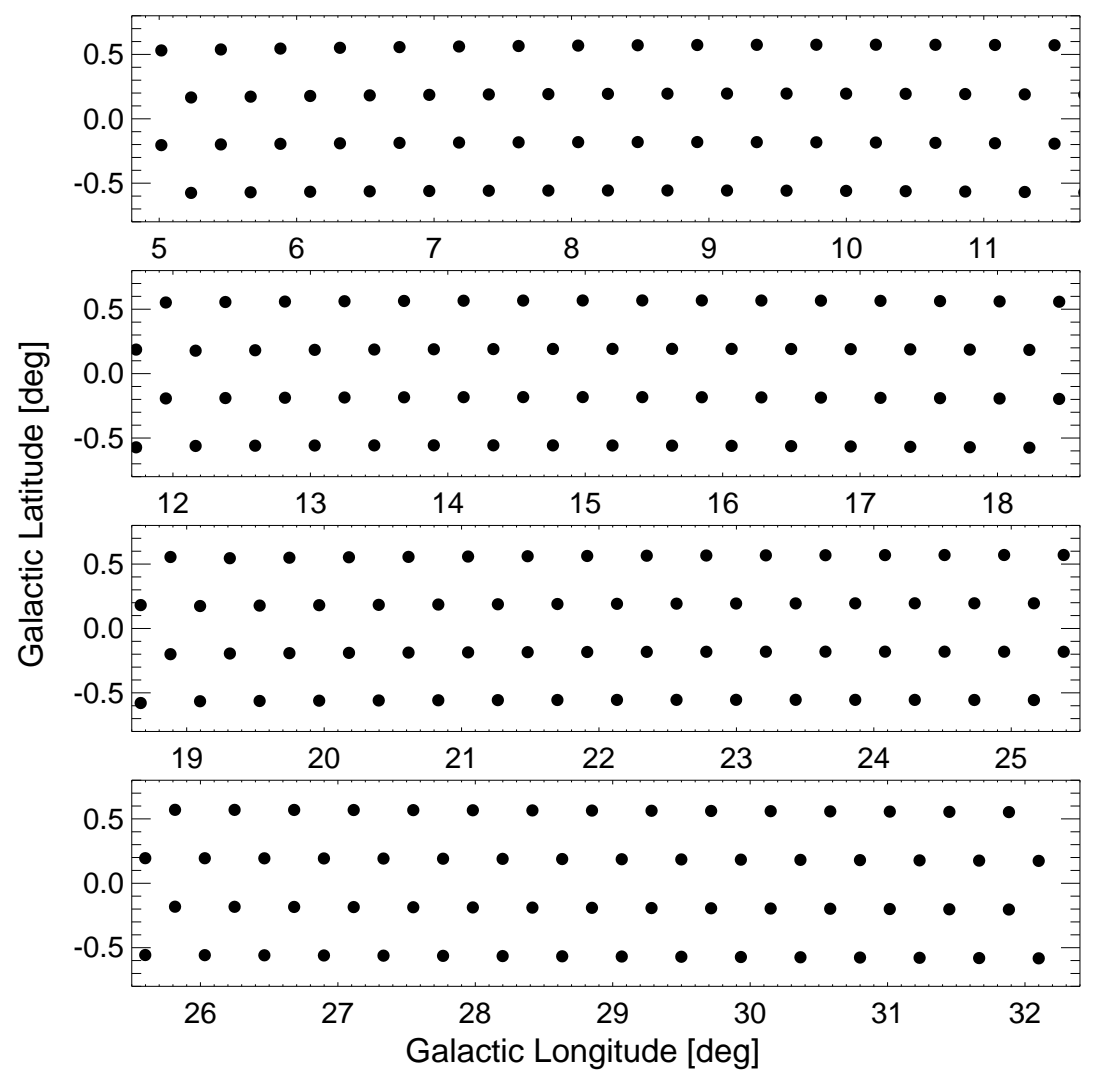

FIG. 1.- The hexagonal grid of 252 VLA pointing centers used for the MAGPIS $20 \mathrm{~cm}$ survey.

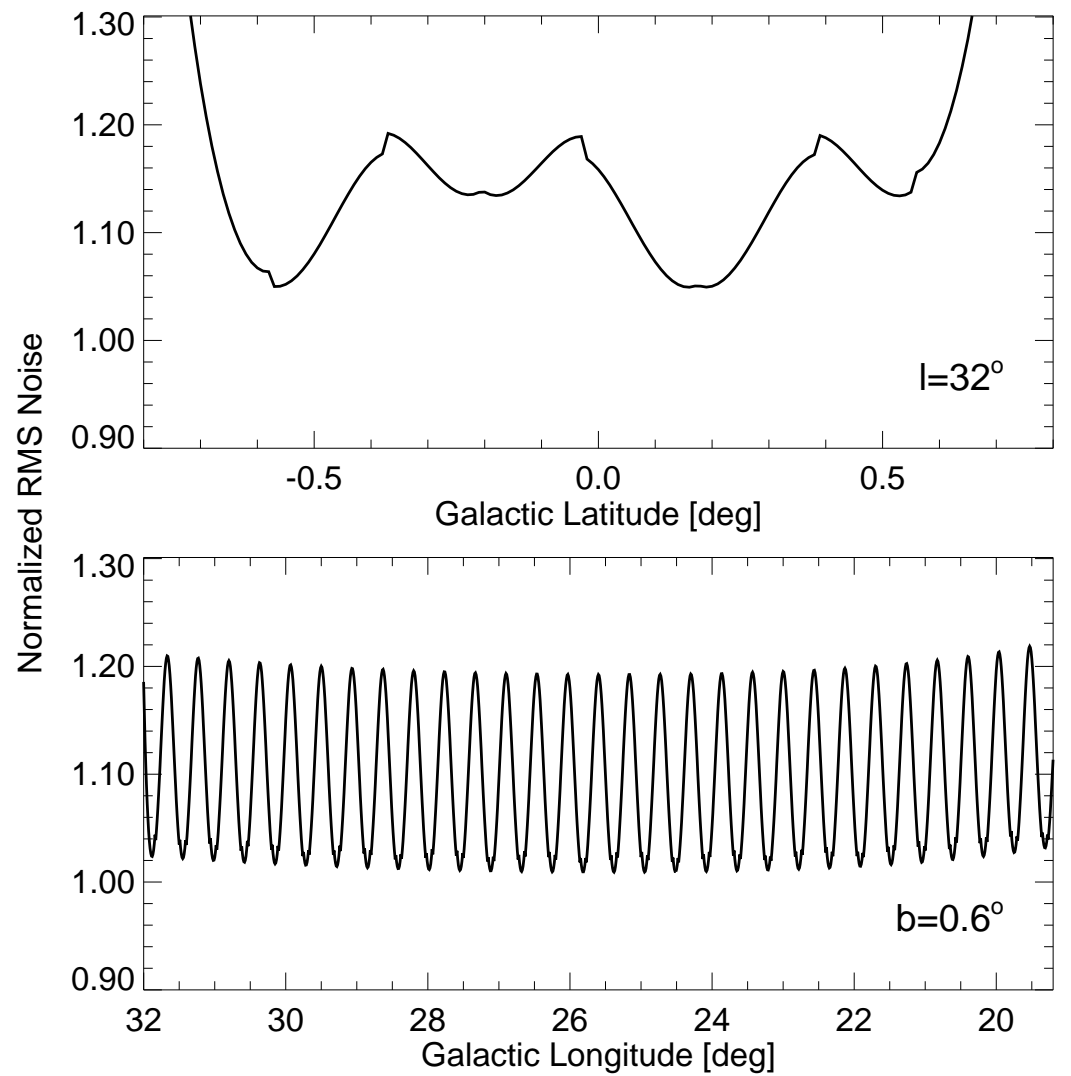

FIG. 2.- The variation in the rms noise as a function of position after the overlapping images have been co-added. The rms is normalized to unity at field center for a single pointing. The top panel shows a cut in latitude at the edge of the survey $\left(l=32^{\circ}\right)$, and the bottom panel shows the rms along a line passing near the field centers at $b=0.6$. The rms noise is uniform, with a peak-to-peak variation of only $\sim \pm 10 \%$ except at the edges of the surveyed area. 
retrieved from the VLA archive $90 \mathrm{~cm}$ data originally taken by Brogan et al. (2005) that cover the remainder of our current survey area $\left(3 .{ }^{\circ} 6<l<20^{\circ},|b|<2^{\circ}\right)$. These data were reduced using a $6^{\prime \prime}$ pixel size and have a resolution of $\sim 25^{\prime \prime}$.

\subsection{The mid-IR data}

We have retrieved the mid-IR images and catalogs of the Midcourse Space Experiment (MSX - Price et al. 2001) from the IPAC database for the regions our survey covers to date. For ease of comparison, we have regridded the E-band $(20 \mu \mathrm{m})$ data onto the same $l, b$ grid used to present the primary MAGPIS images. We have also constructed ratio maps for the $20 \mathrm{~cm}$ and $20 \mu \mathrm{m}$ data for use in separating thermal and nonthermal emission. An example of such an image is displayed in Figure 3. High values of the radio-to-IR ratio generally indicate nonthermal radio emission such as is produced by supernova remnants, while low values tend to highlight dusty $\mathrm{H}$ II regions, although pulsar wind nebulae, dusty old supernova remnant shells, and dust-free H II regions can in principle exhibit intermediate ratios. We defer a quantitative discussion of the comparison of the radio and mid-IR emission to a future paper.

\subsection{The MAGPIS website}

Consistent with our past practice, the raw VLA data on which MAGPIS is based have been available in the VLA archive from the day they were taken. To facilitate use of these data by the broadest possible community, we have constructed the MAGPIS website (http://third.ucllnl.org/gps), which presents our data products in easily accessible forms. In addition to the full-resolution $20 \mathrm{~cm}$ images, the site provides the complementary $90 \mathrm{~cm}$ images, the regridded MSX $20 \mu \mathrm{m}$ images, and an image atlas of diffuse emission regions (see below). The single-configuration 6 and $20 \mathrm{~cm}$ images from our earlier snapshot surveys (White, Becker \& Helfand 2005) are also available. Images can be displayed with userspecified coordinates, box sizes, and intensity scales or can be downloaded as FITS files. The full discrete-source and diffuse catalogs are available for retrieval or through a search query function, as are our catalogs and publications from our earlier snapshot survey work. We expect to add our XMM Xray survey data and the Spitzer GLIMPSE survey images and catalogs as they become available.

\section{THE MAGPIS IMAGES}

In contrast to the extragalactic radio sky, which is rather sparsely populated by mostly compact sources, radio emission in the Galactic plane is dominated by bright, diffuse H II regions and supernova remnants. Thus, the single-snapshot observations and two-dimensional mapping approximations that worked well in the FIRST and NVSS surveys are inadequate for producing high-dynamic-range images for MAGPIS. In this case, the VLA data must be treated as a threedimensional data set. In practice, 3-d distortions scale with offset from the image center; thus, one way to minimize 3$\mathrm{d}$ effects is to tile the VLA's $30^{\prime}$ primary beam with many small images. We have used a grid of 21 by 21 images, each of which is 128 by 128 pixels in size. Our initial data were reduced on a Sun Ultra 60, with each image requiring $\sim 12$ hours to CLEAN. We subsequently migrated the analysis to a dual-processor Pentium 4 computer that is approximately seven times faster. Since the images are greatly improved by self-calibration, each field has to be reprocessed several times.
Even with data from the D configuration, the resulting maps suffer missing flux from large-scale structure $\left(\gg 1^{\prime}\right)$ to which the VLA is insensitive. To correct for this deficiency, we combined the VLA images with images from a $1400 \mathrm{MHz}$ survey made with the Effelsberg 100-m telescope (Reich, Reich, and Fürst 1990) $\left(\sim 9.4^{\prime}\right.$ angular resolution). The AIPS task IMERG makes FFTs of both the VLA and Effelsberg images, combines the derived FFT amplitudes after normalizing to the $u v$ amplitudes in the region of baseline overlap, and then converts back to the image plane to produce the final individual images. We use a 6.' $2 \times 5$ !' 4 restoring beam on maps with a pixel size of 2 !'. The individual maps are ultimately summed and rebinned to produce mosaic images in Galactic coordinates. The dynamic range varies somewhat with location but, measured as a ratio of the peak flux in the brightest source to the full image rms, is typically in excess of 1000:1 in a 1 $\mathrm{deg}^{2}$ image. Over most of the images, $1 \mathrm{mJy}$ point sources are easily detected.

\section{THE MAGPIS CATALOGS}

The large, diffuse emission features and variable background, coupled with source size scales ranging from arcseconds to degrees, render impractical the type of automated source detection algorithms applied to extragalactic radio surveys. Thus, we have employed the human eye-brain detection system to search the 16.7 million MAGPIS beam areas for radio sources. We divided the problem into two parts: the detection and cataloging of discrete objects less than a few beam areas in size and unconfused by extensive diffuse emission, and regions of sky in which significant diffuse emission is present.

\subsection{Discrete source detection}

A square field was defined around each candidate discrete source. In cases where it was impossible to isolate a single emission peak (e.g., for overlapping or closely clustered sources), multiple sources were included in one field and the field was flagged as "multiple". The default field size was $34^{\prime \prime} \times 34^{\prime \prime}$, but this size was adjusted for larger sources (increased), for high density areas (decreased), or for other reasons (increased or decreased on a case by case basis) such as nearby bad pixels, proximity to the edge of the maps, etc. For the entire survey area this process yielded 2628 single-source fields, and 467 multiple-source fields.

The AIPS-compatible task HAPPY - the source detection algorithm developed for the FIRST survey (see White et al. 1997 for details) - was then run on each of the fields. In HAPPY, a local rms level was calculated for each field using an area three times the input field area; a minimum detection level of five times the local rms was set for each field.

Using the HAPPY output, we rejected any source with a fitted peak flux, $F_{p}<1.0 \mathrm{mJy}$ or $\leq 5.0$ times the local rms, whichever is higher. We also rejected any source with a fitted minor axis less than 3 ." $^{\prime \prime}$ (the beam minor axis is 5."4, and experience from the use of HAPPY in the FIRST survey shows that the vast majority of such "skinny" sources are spurious sidelobes); this only eliminated one source that passed the $F_{p}$ and rms criteria. This process yielded a catalog of 3229 sources.

Although restricting HAPPY to predetermined fields around candidate sources should reduce the number of spurious detections, this method is still susceptible to poor fits resulting from complex, extended emission as well as areas of patterned noise near bright sources. To assess these potential 


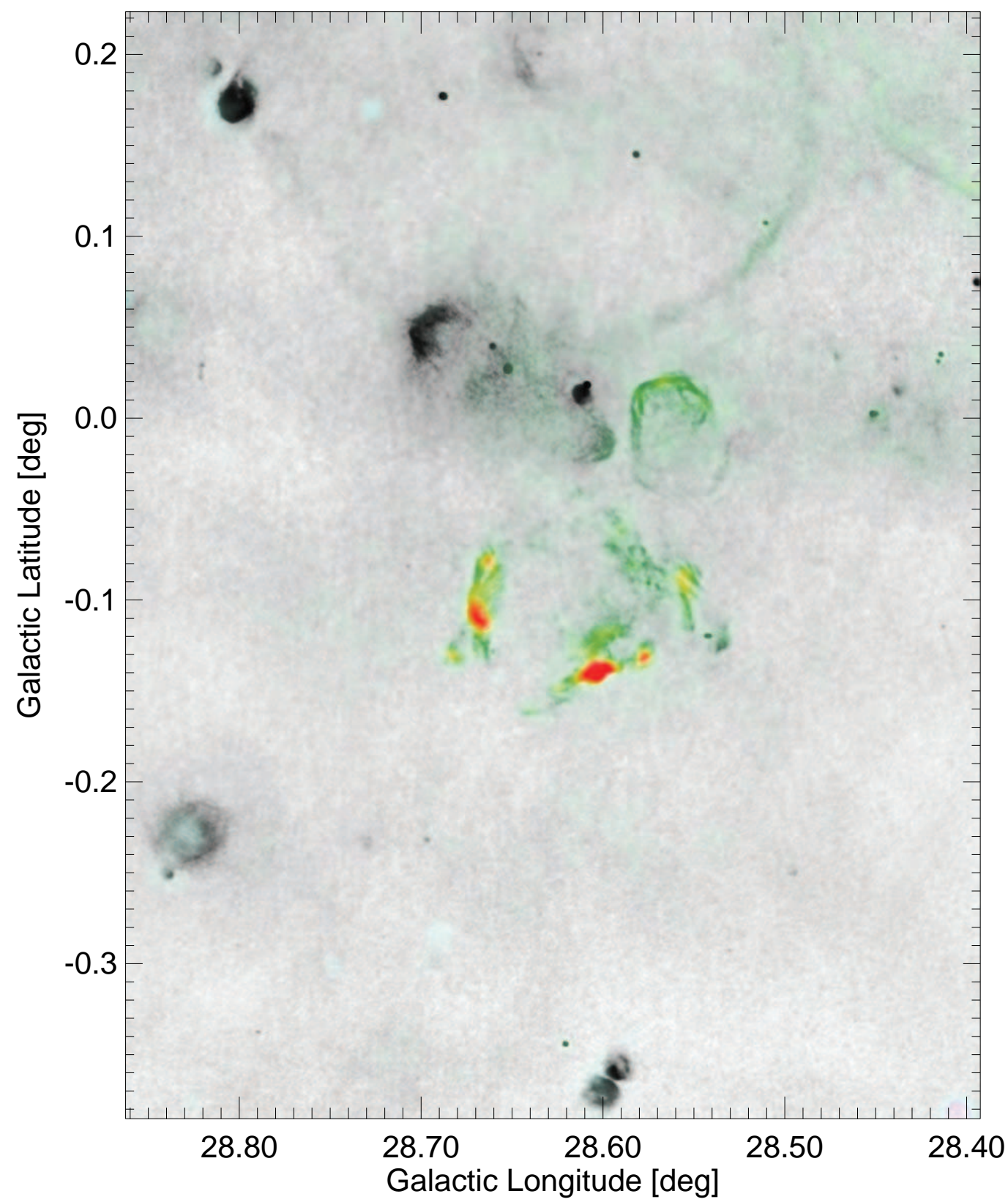

FIG. 3.- Combined radio-IR image demonstrating the separation of thermal and non-thermal emission. The radio image is used to set the intensity of the displayed image, while the radio-IR flux ratio is used to set the color hue and saturation. Objects with strong IR emission (typical of thermal radio sources) appear in black and white, while objects that have absent or weak IR emission (typical of nonthermal radio sources) appear in colors ranging from green to red depending on the upper limit on the radio-to-IR ratio. Both the known SNR G28.6-0.1 and a previously undiscovered remnant at G28.56-0.01 are apparent, as are a half-dozen thermal sources with varying morphologies.

causes of contamination, we flagged for further examination HAPPY solutions

1. when HAPPY fit more than one source in a singlesource input field (67 fields, 144 sources);

2. when HAPPY fit more than two sources in a multiplesource input field (69 fields, 219 sources); or

3. when any fit not meeting (1) or (2) had a major axis $>15^{\prime \prime}$, a major to minor axis ratio greater than 2.0 , or $F_{\text {int }} / F_{p}>5.0$ (136 fields, 143 sources).

In total, 506 fields were flagged and examined. Of these 338 were determined to be good fits, 88 to be "acceptable" fits, 56 to be artifacts or noise, and 24 to be extended rather than discrete sources; the latter were moved to the extended source atlas (see below) and the artifacts were deleted.

The 88 "acceptable" fits are all (in our best judgement) real radio sources, but they are distinguished from good fits in that, upon inspection, it is clear that the two-dimensional elliptical Gaussian employed by HAPPY to extract source parameters is a poor representation of the source surface brightness distribution. We report the HAPPY-derived parameters for these sources in Table 2 for consistency, but flag them accordingly.

The final catalog, presented in Table 2, includes 3149 discrete sources. A Galactic longitude \pm latitude-based name (col. 1) is followed by the peak and integrated flux densities from the Gaussian fit (cols. 2 and 3), with the $S_{i}$ value flagged for the "acceptable" fits described above, and the estimated rms noise level (col. 4). The major and minor axes 
(full-width at half-maximum) and position angle for the elliptical Gaussian complete the morphological description of these compact sources. The last two columns give the infrared $8 \mu \mathrm{m}$ and $21 \mu \mathrm{m}$ flux densities for sources with MSX matches (described further below in $\$ 5.1$ ).

Owing to the variable background and numerous regions of bright diffuse emission, the threshold for discrete source detection varies significantly over the survey region. We can obtain a mean value for the threshold by comparing the source surface density with that of the FIRST survey (Becker et al. 1995). That survey covers $9033 \mathrm{deg}^{2}$ of the extragalactic sky and includes 781,450 sources not flagged as sidelobes, for a mean surface density of $86.54 \mathrm{deg}^{-2}$ at a flux density threshold of $1.0 \mathrm{mJy}^{6}$. The mean source surface density of discrete sources in MAGPIS is $74.8 \mathrm{deg}^{-2}$ considering the entire survey area of $42.1 \mathrm{deg}^{2}$ and $73.0 \mathrm{deg}^{-2}$ in the $35.6 \mathrm{deg}^{2} \mathrm{ly}-$ ing outside regions of diffuse emission; the former value is higher owing to source clustering. Matching this surface density while allowing for several hundred true Galactic sources outside regions of diffuse emission (see \$5.1), we find an effective discrete source threshold of $\sim 1.5 \mathrm{mJy}$ that yields 58.6 extragalactic sources $\mathrm{deg}^{-2}$ in FIRST. Thus, our survey is significantly incomplete between the minimum reported flux density of $1 \mathrm{mJy}$ and $\sim 2 \mathrm{mJy}$, but, over the $85 \%$ of the area outside regions of diffuse emission (see Fig. 4), it is largely complete above this range. Note that a large majority of the discrete radio sources detected even within $1^{\circ}$ of the Galactic plane are extragalactic objects; this is evident from the lack of a strong Galactic latitude dependence of our source counts seen in Figure 5. Observations at other wavelengths are required to identify the Galactic components of the discrete source population.

\subsection{The diffuse source atlas}

The elliptical Gaussians used in fitting the discrete sources are a poor approximation to the surface brightness distributions for nearly all of the more extended radio sources detected in our survey. Furthermore, for sources extended by more than $\sim 60^{\prime \prime}$, our VLA $u v$ coverage is inadequate to derive accurate flux densities, and the addition of the single dish data, while an asset in making images, has unquantifiable effects on derived flux densities. Thus, we again turn to the eye-brain system for identifying diffuse sources and source complexes, and do not attempt to derive accurate flux density measurements for these sources.

The entire survey region was examined by eye, and regions of extended emission were identified and enclosed in square boxes ranging in size from $1 \operatorname{arcmin}^{2}$ to $48^{\prime} \times 48^{\prime}$. In some instances regions are defined by a single coherent source, while in others a complex of diffuse emission regions is included. A total of 398 such regions covering $7.6 \mathrm{deg}^{2}$ were so identified. For each region, the peak flux density, minimum flux density (a proxy for the noise level in the region), total area, and net flux density were recorded; we emphasize that these flux densities are not necessarily accurate reflections of integrated source intensity and, in some regions, include the flux

6 The snapshot images of the FIRST survey require the addition of a 'CLEAN bias' of $0.25 \mathrm{mJy}$ to the measured flux densities. The greater $u v$ coverage achieved in the multi-array, multi-snapshot MAGPIS survey should significantly reduce CLEAN bias, although it is improbable that the bias is zero. Since, however, absolute calibration is unlikely to be accurate to better than $10 \%$ in light of our addition of single-dish data, and none of our scientific projects require flux densities this accurate, we ignore CLEAN bias in this work. density of several related - or possibly unrelated - sources; they provide only a rough guide to source intensities. We have subtracted from the integrated flux density in each region the sum of the flux densities of the discrete sources from Table 2 that fall within the region; a table listing the cataloged discrete sources within each region is available at the MAGPIS website.

In order to estimate the accuracy of our diffuse flux density estimates, we have compared our flux densities for the 25 known supernova remnants in our survey region with those tabulated in Green (2004). We exclude remnants for which the tabulated value is uncertain (listed with a "?" in Green's catalog), as well as those that do not fall completely within our survey coverage. We scale the $1.0 \mathrm{GHz}$ flux densities listed in Green's catalog to our observing frequency of $1.4 \mathrm{GHz}$ using the tabulated spectral indices. We find a good correlation between the flux densities, albeit with an offset that depends on the size of the remnant (Fig. 6). We conclude that the integrated flux densities listed for the diffuse sources typically overestimate the true fluxes of very large sources by factors of two or more due to backgrounds and confusing sources and recommend caution when using them.

The diffuse regions are cataloged in Table 3. A Galactic longitude \pm latitude-based name is found in column 1 . The box size in column 2 and an intensity scaling factor for display purposes (col. 3) precede the brightest pixel value (col. 4) and its location (cols. $5 \& 6$ ), and the integrated flux density inside the box (col. 7). Column 8 provides names for known supernova remnants.

Cleaving to the maxim that quantifies the relative information content of words and pictures, we have constructed a diffuse source atlas to accompany the full survey images on the MAGPIS website. Here, Table 3 is reproduced with active links that allow the user to overlay circles representing sources from the discrete source catalog and contours of the $20 \mu \mathrm{m}$ images from the MSX catalog (Eagen et al. 2003). Each image can also be downloaded as a FITS file.

The website also includes large area $(4.5 \times 1.6)$ JPEG versions of the MAGPIS images with the diffuse region boxes overplotted. It is difficult to display these high-dynamic-range images with a single constrast stretch, and indeed the discrete sources are almost invisible in these images, but they are nonetheless useful for viewing the environment of the diffuse sources.

The MAGPIS discrete source catalog and diffuse source atlas provide an improvement of more than an order of magnitude in both angular resolution and sensitivity over existing Galactic plane survey data. When combined with existing catalogs at other wavelengths along with data from X-ray and infrared surveys currently underway, MAGPIS will provide a resource for studying both thermal and nonthermal processes that mark the evolution of massive stars in the Milky Way. We have a number of followup projects underway; below we briefly comment on the impact the survey is likely to have on our knowledge of the $\mathrm{H}$ II region and supernova remnant populations of the Galaxy.

\section{GALACTIC THERMAL EMISSION REGIONS: MAGPIS AND} MID-IR IMAGES

The critical dependence of an H II region's radio luminosity on the ionizing flux of its exciting star(s) allows for the contruction of a particularly pure census of massive star formation: the $20 \mathrm{~cm}$ radio flux density falls by a factor of 300 between exciting star types 09.5 and B1 such that, at $20 \mathrm{kpc}$, O- 

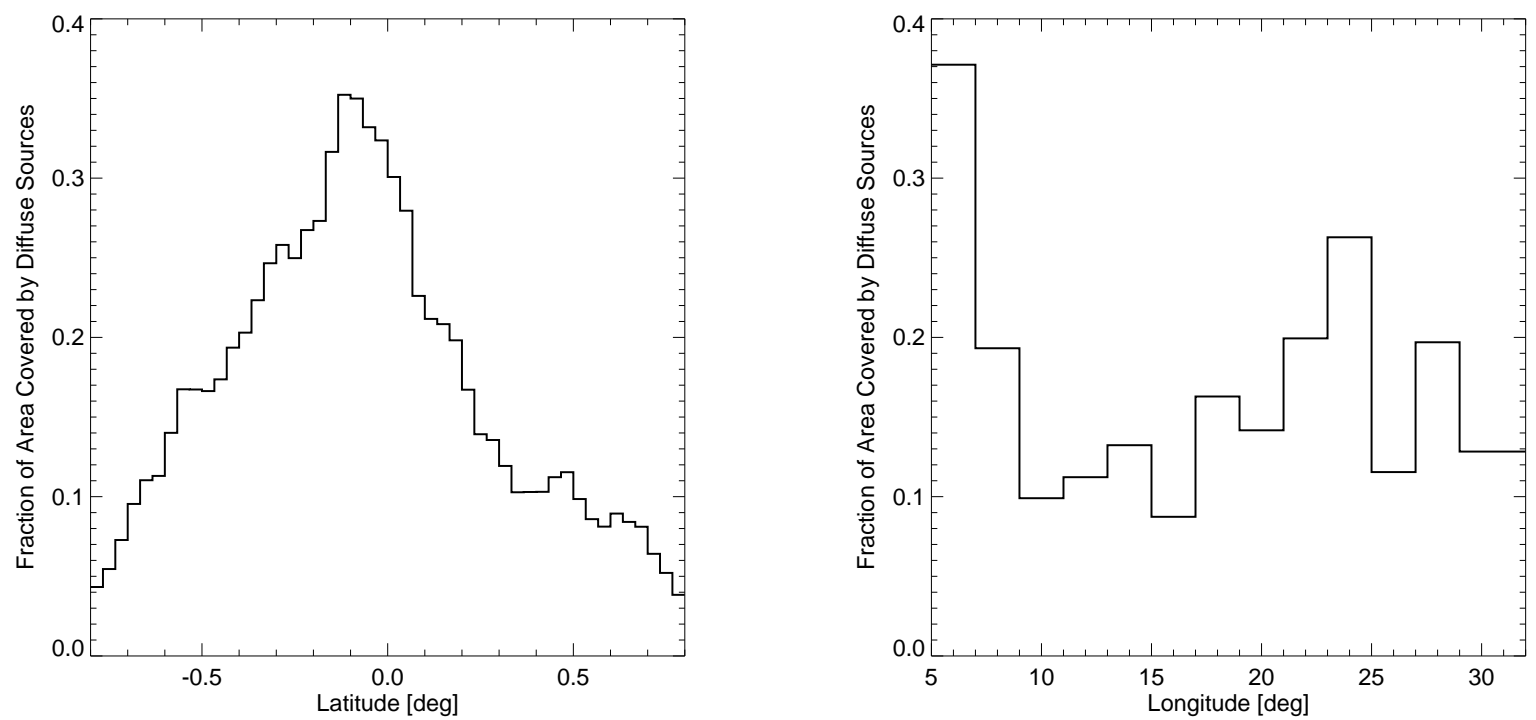

FIG. 4.- The fraction of sky area covered by the diffuse source regions defined in Table 3. These diffuse sources reduce the completeness of our catalogs for faint sources. (a) The distribution with Galactic latitude is strongly concentrated toward the plane, with about $35 \%$ of the area near $b=0^{\circ}$ falling in diffuse source regions. (b) The Galactic longitude distribution is more uniform, though an increase is evident at the survey edge nearest the Galactic center.

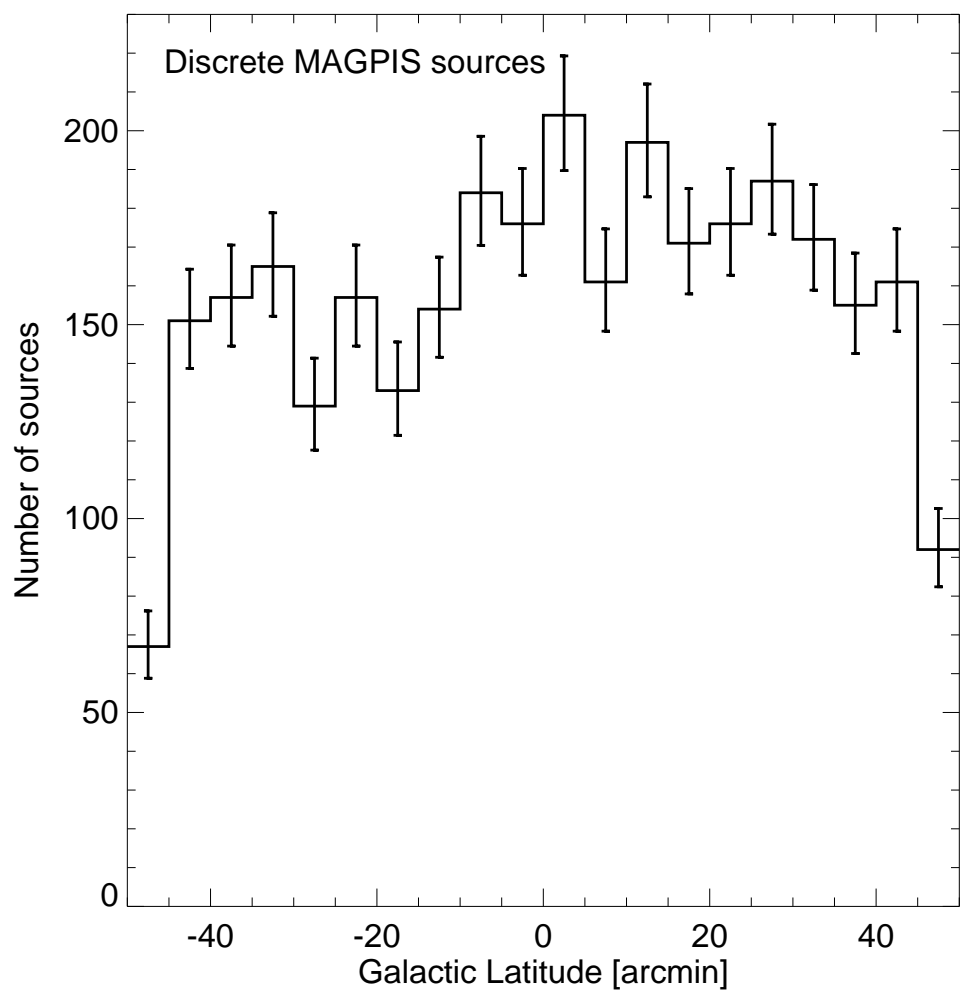

FIG. 5.- Galactic latitude distribution for the discrete source catalog. Even in the Galactic plane the $20 \mathrm{~cm}$ radio sky is dominated by extragalactic sources, so no strong latitude dependence is seen. The counts fall off in the $|b|>45$ arcmin bins due to the drop in sensitivity at the edge of the survey.

star H II regions fall a factor of $>30$ above our survey threshold, while less-massive star-forming complexes (which produce at most B stars) fall a factor $>10$ below it ${ }^{7}$. To separate

7 These numbers are valid only for optically thin nebulae. Compact, optically thick H II regions are self-absorbed at $20 \mathrm{~cm}$ such that only stars earlier than $\mathrm{O} 7$ would fall above our threshold at the far side of the Galaxy (see Table 1 of Giveon et al. $2005 \mathrm{~b}$ for details). Our old $6 \mathrm{~cm}$ snapshot survey allows us to find these sources down to spectral type O9.5; see Giveon et al. (2005a). the $\mathrm{H}$ II regions from the more numerous extragalactic source populations and the extended regions of Galactic nonthermal emission requires observations at another wavelength. Our $6 \mathrm{~cm}$ snapshot survey is useful for the most compact sources $\left(D<15^{\prime \prime}\right)$ but resolves out flux on larger scales. Since most $\mathrm{H}$ II regions contain dust that is heated by the stellar radiation, the mid-IR also can serve as a useful discrimnant.

Figure 7 shows several examples of $\mathrm{H}$ II complexes from 


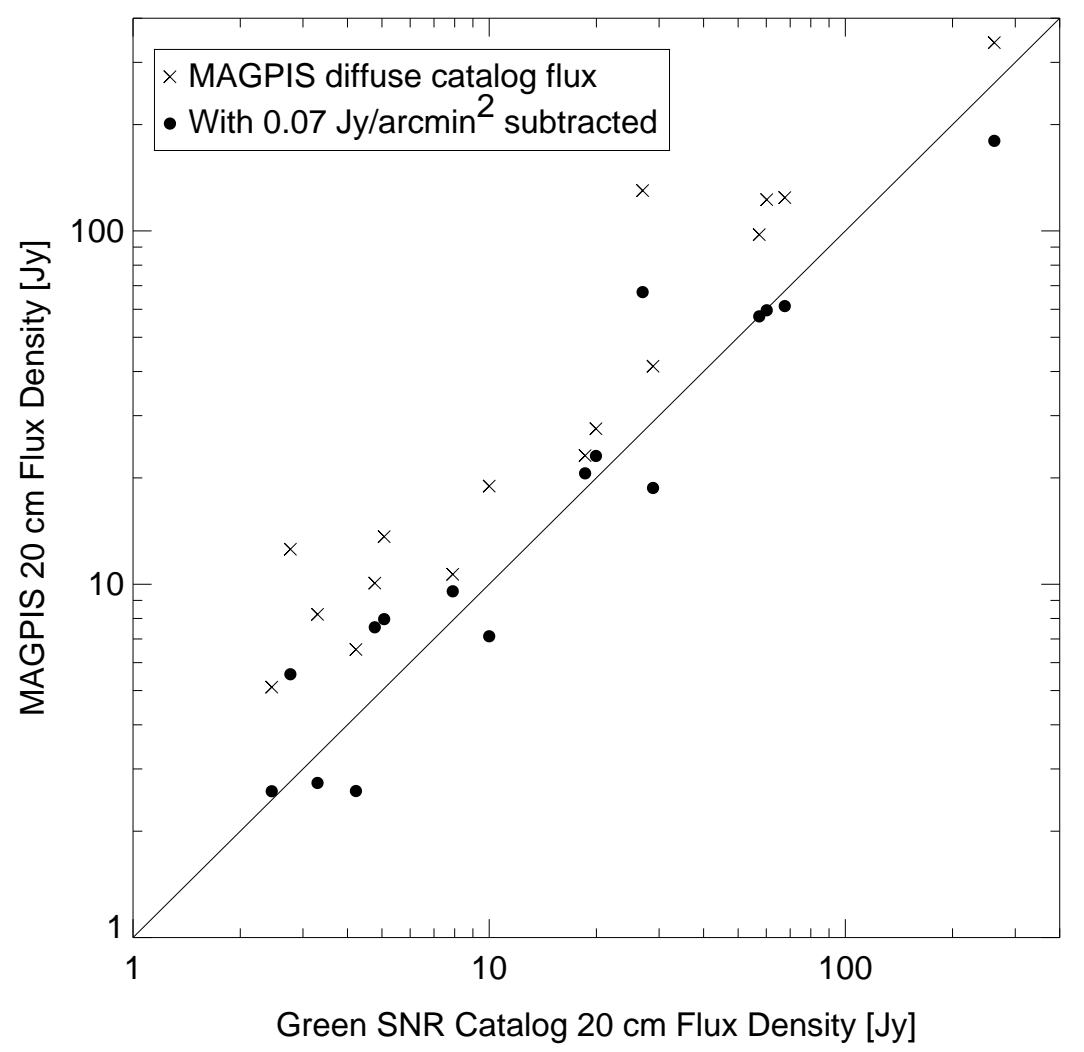

FIG. 6.- Flux densities from our diffuse source catalog for supernova remnants from the Green (2004) catalog. The $\mathrm{x}$ symbols show that our catalog flux densities for these extended sources are typically higher than the Green values by factors of two or more. Subtracting a diffuse backgroun of $0.07 \mathrm{Jy} / \mathrm{arcmin}^{2}$ from our flux densities improves the agreement (dots). This offset most likely arises from a combination of a) absolute calibration differences between the Effelsberg and VLA data, b) the zero-point choice of the former, c) imperfect matching of the $u v$ data (see above), d) the nonlinear nature in which the two datasets are combined into an image via the use of CLEAN, and e) the subjective choice of areas over which to integrate the flux density of individual remnants.

our radio survey with contours from the MSX $20 \mu$ m images overlaid. The degree of correspondence is remarkably good and provides a straightforward method for separating thermal and nonthermal emission in star formation regions. On the MAGPIS website we also provide large-scale radio images with boxes marking the previously published H II regions collected in the Paladini et al. (2003) meta-catalog. It is clear that the MAGPIS data (along with other radio and IR surveys) will enable the construction of a vastly improved H II region cata$\log$.

We defer a detailed analysis of the $\mathrm{H}$ II region population to a future paper; here we provide some simple statistics for compact and ultracompact H II regions by matching our discrete source data to the MSX catalogs as an indication of the wealth of information such a comparison contains. The higher-resolution and greater sensitivity of the Spitzer GLIMPSE data soon to become available will fill in the $2-8 \mu \mathrm{m}$ band and provide crucial information in the most crowded regions.

\subsection{Match to the MSX $20 \mu \mathrm{m}$ catalog}

The $20 \mathrm{~cm}$ survey region is completely covered by the MSXPSCv2.3 "MSX6C" (Egan et al 2003) data set. We searched for MSX6C sources using a search radius of $12^{\prime \prime}$ around each of the discrete $20 \mathrm{~cm}$ sources. To be accepted as a match, the MSX6C source was required to have a quality flag of 2 in at least one of the four bands (see Lumsden et al. 2002). If more than one MSX6C source fell within the search radius for a single $20 \mathrm{~cm}$ source, the MSX6C source closest to the $20 \mathrm{~cm}$ source was kept (this only occurred once). A total of $376 \mathrm{MSX6C}$ sources corresponding to $41820 \mathrm{~cm}$ sources were matched in this manner.

To estimate the number of false matches we repeated the matching process using fake catalogs produced by shifting the MSX6C catalog $\pm 10^{\prime}$ and $\pm 20^{\prime}$ in longitude. Since, for example, the vast majority (78\%) of the MSX sources are stars detected only in the $8 \mu \mathrm{m}$ band (very few of which have radio counterparts), we can greatly reduce the false match rate by assessing the false rates separately for sources detected in different band combinations. We have followed the methology descibed in Giveon et al. (2004; see also White et al. 1991), to arrive at a false-match reliability criterion for each of the band combinations in which a $20 \mathrm{~cm}-\mathrm{MSX} 6 \mathrm{C}$ match existed. Using a reliability of $R>90 \%{ }^{8}$ we find 245 MSX6C sources (of which $\sim 8$ should be false) matched to $27820 \mathrm{~cm}$ sources. Of these, 217 are single $20 \mathrm{~cm}$-MSX6C matches, 23 are cases in which one MSX6C source matches two $20 \mathrm{~cm}$ sources, and 5 represent one MSX6C source matching three $20 \mathrm{~cm}$ sources.

The distribution of the matched and unmatched sources on the sky is displayed in Figure 8. While sources with infrared matches are found throughout the latitude coverage, it is clear that they concentrate toward the plane. The latitude distribution is displayed in Figure 9. The distribution peaks at $b=0^{\circ}$ with a surface density of 22 sources $\mathrm{deg}^{-2}$ (when regions obscured by bright diffuse sources are excluded), and has a full width half maximumn of less than $15^{\prime}$. Examination of the

\footnotetext{
8 This eliminates MSX sources detected only in the $8 \mu \mathrm{m}$ band as well as those detected in $8 \mu \mathrm{m}$ and $12 \mu \mathrm{m}$ only, and $8 \mu \mathrm{m}, 12 \mu \mathrm{m}$, and $14 \mu \mathrm{m}$ only. This removes 131 sources (67\% of which are false matches), leading to a catalog of matches that is $>95 \%$ reliable and $\sim 90 \%$ complete.
} 

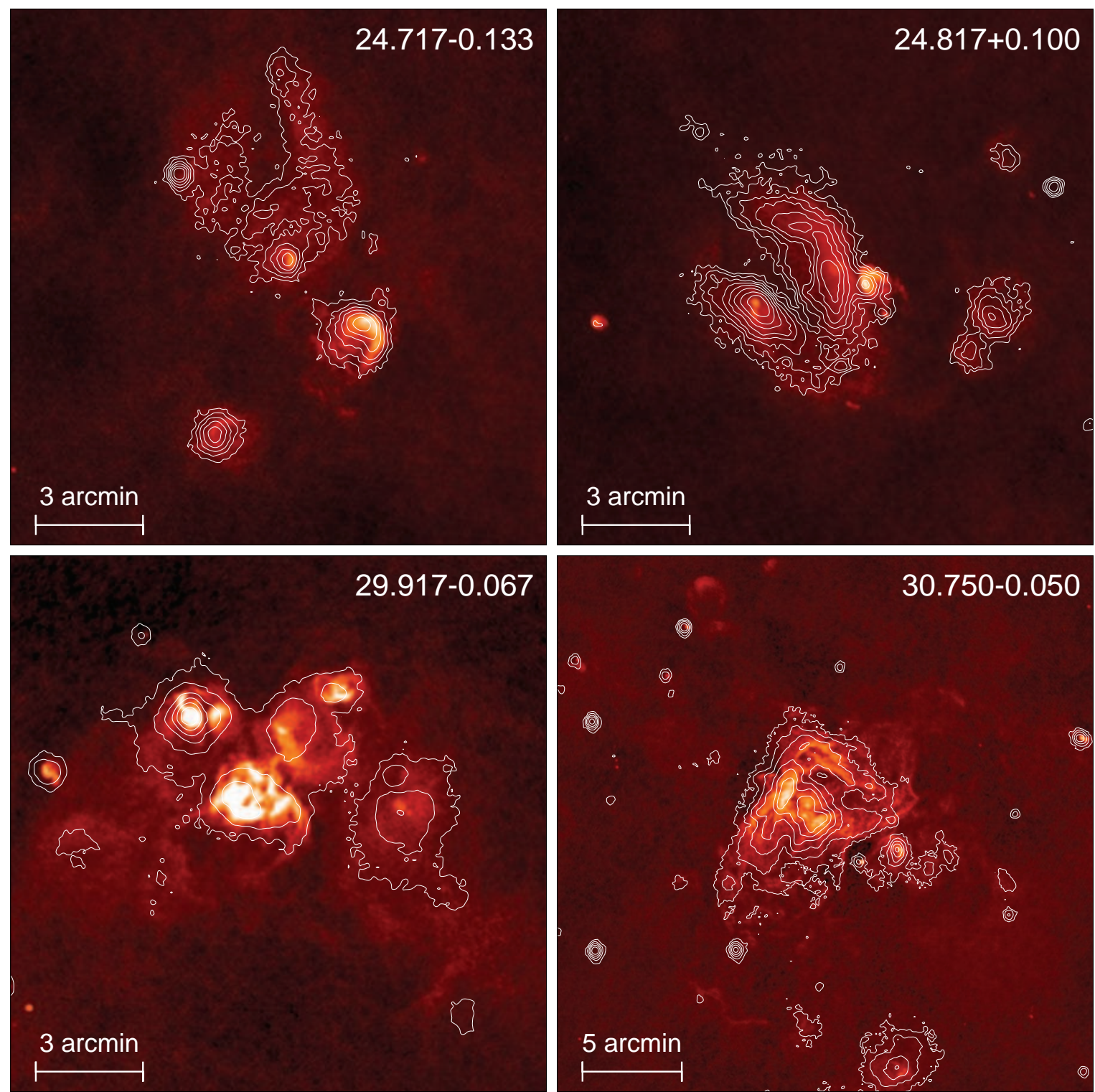

FIG. 7.- Examples of H II complexes from our radio survey with the MSX $20 \mu \mathrm{m}$ image contours overlaid, showing the excellent radio-IR correspondence for these thermal sources.

atlas of extended emission shows that there are more thermal sources inside the $6.5 \mathrm{deg}^{2}$ subsumed by the atlas images than outside, and most of these sources are not included in the discrete source catalog. We estimate that there are a total of more than 600 distinct $\mathrm{H}$ II regions in our $42 \mathrm{deg}^{2}$ survey area, although we defer to a future publication the development of a detailed catalog and its analysis.

\section{GALACTIC NONTHERMAL EMISSION IN MAGPIS}

Supernova remnants (SNRs) are among the brightest radio sources - and the brightest X-ray sources - in the Galaxy. They are a dominant source of mechanical energy input to the ISM, drive the Galaxy's chemical evolution, and mark the birthsites of neutron stars and black holes. Yet our knowledge of the Galactic population is woefully incomplete, owing to the low angular resolution of previous radio and hard X-ray surveys of the plane, and the soft spectral response of previous $\mathrm{X}$-ray imaging observations. A total of 231 remnants appears in the latest catalog (Green 2004); Brogan et al. (2004) have recently added three new remnants in one of our fields. The current rate of discovery is a few remnants per year. However, based on 1) extragalactic SN rates ( $\sim 1-2$ per century) combined with SNR lifetimes $\left(2.5-5 \times 10^{4} \mathrm{yr}\right)$, and 2) a detailed analysis of the current SNR distribution (Helfand et al. 1989), we expect the total population to be between 500 and 1000 . The youngest remnant we know is 340 years old; four to seven younger ones exist somewhere in the Galaxy.

MAGPIS can detect pulsar-driven remnants to a luminosity $10^{-4}$ that of the Crab Nebula at the edge of the Galaxy (or $\sim 10 \%$ that of 3C58, the least luminous young Crab-like remnant known). For shell-like SNRs, our survey will be sensitive to all young remnants. For example, we will detect remnants throughout the surveyed volume down to luminosities $0.01 \%$ that of Cas A, and can even see a clone of the underluminous historical remnant SN 1006 at $20 \mathrm{kpc}$ : it would appear as a 1'diameter source with a flux density of $\sim 25 \mathrm{mJy}$. Our survey could detect a remnant equivalent to SN1987A from the time it was 3 years old anywhere in the survey region, and would resolve such a remnant only 15 years after the explosion.

Twenty-five known remnants fall within the current survey area, and all are easily detected. The known remnants are indicated in Table 3; in many instances, the maps presented here 


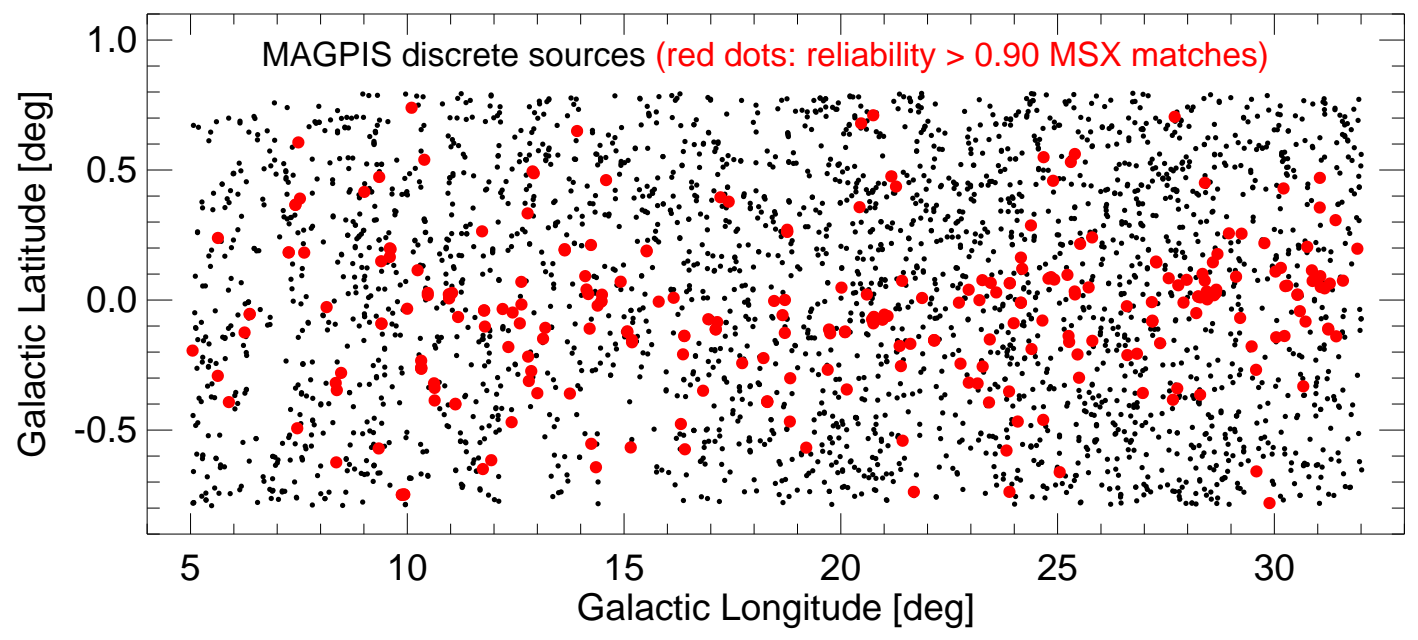

FIG. 8.- Sky distribution of MAGPIS $20 \mathrm{~cm}$ radio sources from the discrete source catalog. Red dots show sources with confident (reliability $>0.90$ ) infrared counterparts in the MSX catalog. The radio-MSX matches are clearly concentrated toward the plane.

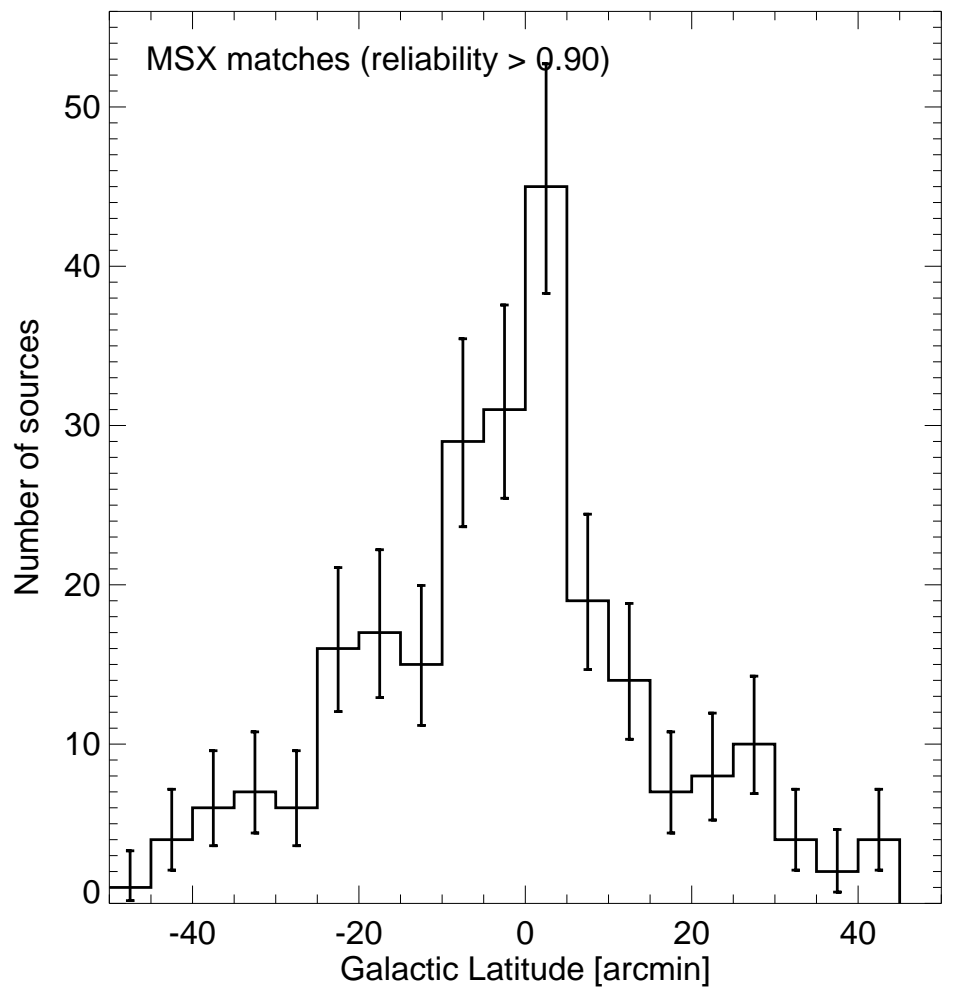

FIG. 9.- Galactic latitude distribution for the 245 MSX sources having reliable radio matches. These sources are highly concentrated toward the plane of the Galactic disk.

are the best available. Images for the eleven remnants smaller than $10^{\prime}$ in diameter — most of which lack high-resolution maps in the literature - are displayed in Figure 10.

As can be seen by browsing the diffuse-source atlas, there are a large number of shell-like sources detected in our survey. Without observations at other wavelengths, however, it is impossible to separate the thermal and non-thermal sources to derive a list of new SNR candidates. Fortunately, as noted above, we do have VLA data covering the entire region at $90 \mathrm{~cm}$, as well as the MSX mid-IR images. A simple qual- itative comparison of these three datasets (available for the reader at the MAGPIS website) allows us to identify quickly high-probablity SNR candidates.

In Table 4, we present 49 new SNR candidates in our $27^{\circ}$ slice of Galactic longitude. To derive this list, we have required:

- the object has a very high ratio of $20 \mathrm{~cm}$ to $20 \mu \mathrm{m}$ flux (i.e., it is typically undetectable in the $20 \mu \mathrm{m}$ MSX image); 

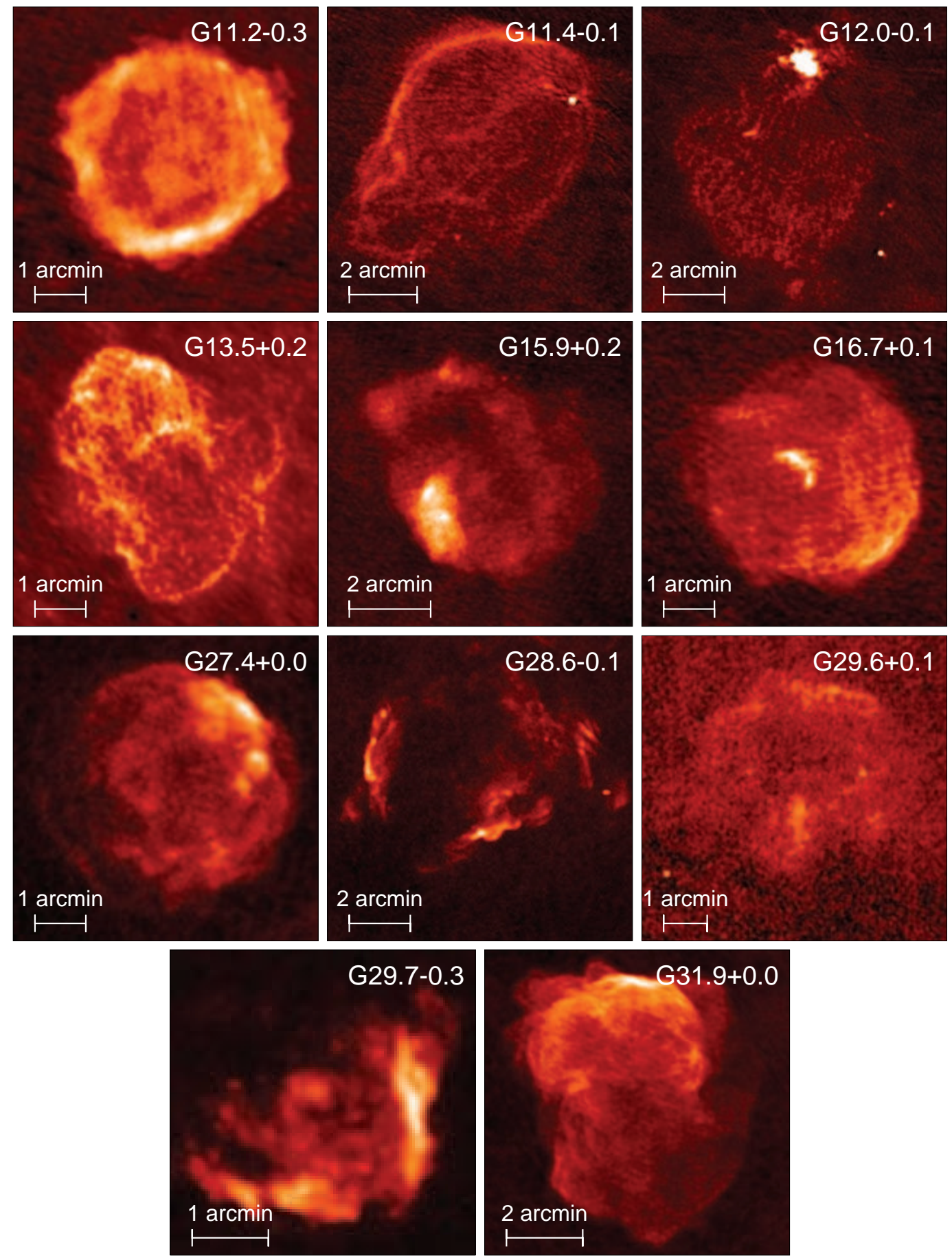

FIG. 10.- MAGPIS $20 \mathrm{~cm}$ images of the eleven previously known supernova remnants in the survey area with diameters less than $10^{\prime}$

- the object has a counterpart in our $90 \mathrm{~cm}$ images with a similar morphology and a higher peak flux density; and

- the object has a distinctive SNR morphology. For shelltype remnants we require at least half of a complete shell, while for the two pulsar wind nebula candidates we see a centrally peaked brightness distribution.

For most of these candidates the data in columns 1, 3, 4 and 5 are repeated from Table 3 . Column 2 gives the source diameter (as opposed to the display box size in column 3, which is always larger). Five of the the entries in this table are components of larger sources listed in Table 3, with three associated with the large diffuse complex at G19.60-0.20 and two associated with G6.50-0.48.

Images for a dozen candidates ranging in size from $40^{\prime \prime}$ to $9^{\prime}$ are displayed in Figure 11. Not unexpectedly, the diameter distribution for our remnant candidates varies markedly from that of the known remnant population. Assuming that followup spectral and polarimetric observations confirm the large majority of these sources as SNRs, we will have tripled the number of known remnants in this region of the Galaxy. However, while the number of remnants with $D \geq 10^{\prime}$ will only rise from 13 to 16 , the number with $10^{\prime}>D \geq 5^{\prime}$ will quadruple from 5 to 19 , while the number with $D<5^{\prime}$ will 

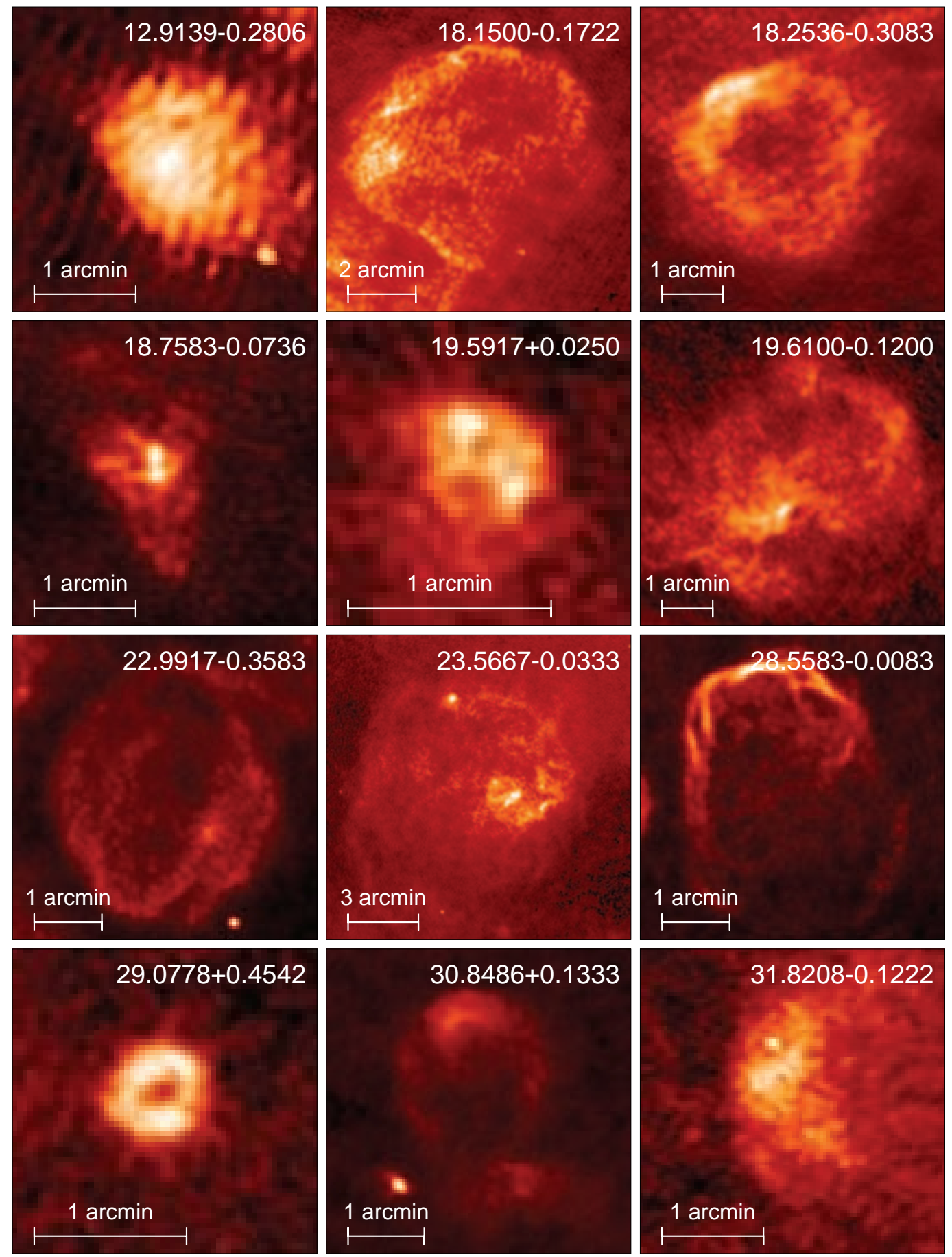

FIG. 11.- MAGPIS $20 \mathrm{~cm}$ images of 12 new supernova remnant candidates.

rise more than sevenfold from 5 to 37 .

Particularly interesting among these new SNR candidates are those that may harbor young, high- $\dot{E}$ pulsars. In addition to the two PWN candidates, there are two shell-like remnants with central diffuse emission peaks highly reminiscent of composite SNRs. Given the core-collapse SN rate in the Galaxy, we should expect to find $\sim 10$ neutron stars younger than the Crab and 3C58 pulsars (Kaspi and Helfand 2002). While these new sources are significantly dimmer than even the underluminous PWN 3C58 (Bietenholz, Kassim, and Weiler 2001; Slane et al. 2004), if they are at distances of $\sim 15 \mathrm{kpc}$, their luminosities are comparable. Also notewor- thy are the three shell-type remnants with diameters less than $1^{\prime}$. At $15 \mathrm{kpc}$, their diameters are $\sim 3 \mathrm{pc}$, corresponding to an age of $\sim 130$ years for a radio expansion rate comparable to that of SN1987A e.g., Eli et al. 2003).

The SNR candidates listed in Table 4 far from exhaust the nonthermal emission features in our survey area; a roughly comparable number of filaments and arcs with apparently nonthermal radio spectra and no IR counterparts are seen. Furthermore, there are several regions in which thermal and nonthermal features are cospatial; these will require scaledarray observations at several frequencies to disentangle. Nontheless, it is clear that high-dynamic-range, high-sensitivity 
observations of the type reported here are essential for characterizing fully the Galactic SNR population.

\section{SUMMARY AND FUTURE PROSPECTS}

We have presented a $6^{\prime \prime}$ resolution centimetric image of the plane of the Milky Way in a portion of the first quadrant that represents an improvement over existing surveys by more than an order of magnitude in resolution, sensitivity, and dynamic range. The survey detection threshold is 1 to $2 \mathrm{mJy}$ over most of the survey area. We identify over 3000 discrete radio sources and $\sim 400$ regions of diffuse emission, presenting catalogs and atlases that quantify the source properties. We include complementary $90 \mathrm{~cm}$ images over the entire survey region and provide a comparison with mid-IR data; taken together, these latter two datasets help to separate thermal from nonthermal emssion regions. We find several hundred H II regions in the survey area, many reported here for the first time. We also identify 49 high-probability supernova remnant candidates, including a seven-fold increase in the number of remnants with diameters smaller than $5^{\prime}$ in the survey region. All of the survey's results are available at the MAGPIS website.

Considerable work remains to exploit fully the survey results. A complementary hard X-ray survey over portions of this region is being conducted with XMM-Newton, and sev- eral followup obervations of interesting sources are scheduled with XMM and Chandra. Scaled-array polarimetric and photometric observations with the VLA are required to confirm the SNR candidates. As the Spitzer GLIMPSE program images become available, further progress will be possible in identifying compact and ultra-compact $\mathrm{H}$ II regions and in using these to provide a census of the OB star population; higher frequency observations with the VLA will be required to identify optically thick H II regions. Future observations to extend the MAGPIS coverage area will provide the basis for a comprehensive view of massive star birth and death in the Milky Way.

DJH and RHB acknowledge the support of the National Science Foundation under grants AST-05-07598 and AST-026-55; DJH was also supported in this work by NASA grant NAG5-13062. RHB's work was supported in part under the auspices of the US Department of Energy by Lawrence Livermore National Laboratory under contract W-7405-ENG-48. RLW acknowledges the support of the Space Telescope Science Institute, which is operated by the Association of Universities for Research in Astronomy under NASA contract NAS5-26555.

\section{REFERENCES}

Becker, R. H., White, R. L., McLean, B. J., Helfand, D. J. \& Zoonematkermani, S. 1990, ApJ358, 485

Becker, R. H., White, R. L., Helfand, D. J. \& Zoonematkermani, S. 1994, ApJS91, 347

Becker, R. H., White, R. L., \& Helfand, D. J. 1995, ApJ450, 559

Benjamin, R. A., et al. 2003, PASP, 115, 953

Brogan, C. L., Devine, K. E., Lazio, T. J., Kassim, N. E., Tam, C. R., Brisken, W. F., Dyer, K. K., \& Roberts, M. S. E. 2004, AJ, 127, 355

Brogan, C. L., Gaensler, B. M., Gelfand, Y., Roberts, M. E. E., Lazio, T. J., \& Kassim, N. E. 2005, X-Ray and Radio Connections (eds. L.O. Sjouwerman and K.K Dyer) Published electronically by NRAO, http://www.aoc.nrao.edu/events/xraydio

Bietenholz, M. F., Kassim, N. E., \& Weiler, K. W. 2001, ApJ, 560, 772

Egan, M. P., et al. 2003, VizieR Online Data Catalog, 5114, 0

English, J., et al. 1998, Publications of the Astronomical Society of Australia, 15,56

Giveon, U., Becker, R. H., Helfand, D. J., \& White, R. L. 2005a, AJ, 129, 348

Giveon, U., Becker, R. H., Helfand, D. J., \& White, R. L. 2005b, AJ, 130, 156

Green, D. A. 2004, Bulletin of the Astronomical Society of India, 32, 335

Hands, A. D. P., Warwick, R. S., Watson, M. G., \& Helfand, D. J. 2004, MNRAS, 351, 31

Helfand, D. J., Velusamy, T., Becker, R. H., \& Lockman, F. J. 1989, ApJ, 341, 151

Helfand, D. J., Zoonematkermani, S., Becker, R. H. \& White, R. L. 1992 ApJS80, 211

Kaspi, V. M., \& Helfand, D. J. 2002, ASP Conf. Ser. 271: Neutron Stars in Supernova Remnants, 271, 3
Lumsden, S. L., Hoare, M. G., Oudmaijer, R. D., \& Richards, D. 2002, MNRAS, 336, 621

McClure-Griffiths, N. M., Green, A. J., Dickey, J. M., Gaensler, B. M., Haynes, R. F., \& Wieringa, M. H. 2001, ApJ, 551, 394

Michael, E., et al. 2003, ApJ, 593, 809

Paladini, R., Burigana, C., Davies, R. D., Maino, D., Bersanelli, M., Cappellini, B., Platania, P., \& Smoot, G. 2003, A\&A, 397, 213

Price, S. D., Egan, M. P., Carey, S. J., Mizuno, D. R., \& Kuchar, T. A. 2001, AJ, 121, 2819

Reed, B. C. 2000, AJ, 120, 314

Reich, W., Reich, P., \& Fuerst, E. 1990, A\&AS, 83, 539

Slane, P., Helfand, D. J., van der Swaluw, E., \& Murray, S. S. 2004, ApJ, 616, 403

Taylor, A.R., Stil, J.M., McClure-Griffiths, N.M., Martin, P.G., Rothwell, T.A., \& Lockman, F.J. 2002 in ASP Conf. Ser. 276,"Seeing through the Dust: The Detection of HI and the Exploration of the ISM in Galaxies, ed. A.R. Taylor, T.L. Landecker, \& A.G. Wells (San Francisco:ASP), 68 White, R. L., Becker, R. H. \& Helfand, D. J. 1991 ApJ371, 148

White, R. L.,Becker, R. H., Helfand, D. J. \& Gregg, M. D. 1997, ApJ475, 479

White, R. L., Becker, R. H., \& Helfand, D. J. 2005, AJ, 130, 586

Zoonematkermani, S., Helfand, D. J., Becker, R. H., White, R. L. \& Perley, R. A., 1990, ApJS74, 181

This work was performed under the auspices of the U.S. Department of Energy by University of California, Lawrence Livermore National Laboratory under contract W-7405-Eng-48. 
Helfand et al.

TABLE 2

CATAlog of Discrete Sources

\begin{tabular}{|c|c|c|c|c|c|c|c|c|}
\hline $\begin{array}{c}\text { Name }(l+b) \\
\text { (1) }\end{array}$ & $\begin{array}{c}S_{p}(20 \mathrm{~cm})^{\mathrm{a}} \\
\text { mJy/beam } \\
(2)\end{array}$ & $\begin{array}{c}S_{i}(20 \mathrm{~cm})^{\mathrm{b}} \\
\text { mJy } \\
(3)\end{array}$ & $\begin{array}{l}\text { RMS } \\
\text { mJy } \\
(4)\end{array}$ & $\begin{array}{c}\text { Major }^{\mathrm{c}} \\
(5)\end{array}$ & $\begin{array}{c}\operatorname{Minor}^{\mathrm{c}} \\
\quad(6)\end{array}$ & $\begin{array}{c}\theta \\
\circ \\
(7)\end{array}$ & $\begin{array}{c}\mathrm{S}(8 \mu \mathrm{m})^{\mathrm{d}} \\
\mathrm{Jy} \\
(8)\end{array}$ & $\begin{array}{c}\mathrm{S}(21 \mu \mathrm{m})^{\mathrm{d}} \\
\mathrm{Jy} \\
(9)\end{array}$ \\
\hline $10.74968-0.41783$ & 1.69 & 7.07 & 0.29 & 15.1 & 9.2 & 54 & $\ldots$ & $\ldots$ \\
\hline $10.78586-0.14376$ & 12.86 & 15.14 & 0.22 & 6.7 & 5.9 & 37 & $\ldots$ & $\ldots$ \\
\hline $10.80070+0.15285$ & 5.28 & 6.51 & 0.16 & 6.8 & 6.1 & 18 & $\ldots$ & $\ldots$ \\
\hline $10.81109+0.75435$ & 1.92 & 2.49 & 0.29 & 8.5 & 5.1 & 83 & $\ldots$ & $\ldots$ \\
\hline $10.82677-0.01064$ & 3.98 & $26.58 \mathrm{E}$ & 0.28 & 17.5 & 12.8 & 16 & 1.10 & 1.56 \\
\hline $10.82960-0.01145$ & 2.05 & $6.68 \mathrm{E}$ & 0.28 & 15.6 & 7.0 & 49 & $\ldots$ & $\ldots$ \\
\hline $10.85201-0.44069$ & 6.61 & 9.99 & 0.22 & 7.3 & 6.9 & 116 & $\ldots$ & $\ldots$ \\
\hline $10.86448-0.32989$ & 2.32 & 3.28 & 0.37 & 7.3 & 6.5 & 60 & $\ldots$ & $\ldots$ \\
\hline $10.89153+0.09971$ & 3.67 & 4.39 & 0.28 & 7.2 & 5.6 & 176 & $\ldots$ & $\ldots$ \\
\hline $10.89487+0.06428$ & 4.00 & 4.93 & 0.39 & 6.8 & 6.0 & 1 & $\ldots$ & $\ldots$ \\
\hline $10.89530+0.19694$ & 1.62 & 4.32 & 0.18 & 12.6 & 7.1 & 54 & $\ldots$ & $\ldots$ \\
\hline $10.89687+0.19994$ & 4.04 & 6.13 & 0.18 & 7.6 & 6.7 & 32 & $\ldots$ & $\ldots$ \\
\hline $10.89866+0.20285$ & 1.19 & 3.28 & 0.18 & 11.3 & 8.2 & 117 & $\ldots$ & $\ldots$ \\
\hline $10.91317+0.23448$ & 30.65 & 51.38 & 0.21 & 8.2 & 6.9 & 138 & 0.16 & $<1.34$ \\
\hline $10.91841-0.48325$ & 1.36 & 2.54 & 0.22 & 9.9 & 6.3 & 9 & $\ldots$ & $\ldots$ \\
\hline $10.92542+0.02681$ & 69.34 & 83.74 & 0.31 & 6.6 & 6.2 & 32 & $\ldots$ & $\ldots$ \\
\hline $10.93848+0.41073$ & 2.58 & 2.93 & 0.20 & 6.9 & 5.5 & 42 & $\ldots$ & $\ldots$ \\
\hline $10.94310-0.40329$ & 5.73 & 22.67 & 0.26 & 12.4 & 10.6 & 94 & $\ldots$ & $\ldots$ \\
\hline $10.94413+0.63106$ & 4.33 & 5.63 & 0.19 & 6.8 & 6.4 & 3 & $\ldots$ & $\ldots$ \\
\hline $10.95074+0.23027$ & 1.78 & 2.28 & 0.24 & 7.1 & 6.0 & 156 & $\ldots$ & $\ldots$ \\
\hline $10.95235-0.48679$ & 1.17 & 2.45 & 0.22 & 9.9 & 7.1 & 78 & $\ldots$ & $\ldots$ \\
\hline $10.95854+0.02233$ & 37.32 & 47.92 & 0.40 & 6.8 & 6.3 & 28 & 0.35 & 12.58 \\
\hline $10.96456+0.00641$ & 24.92 & $196.93 \mathrm{E}$ & 0.37 & 23.7 & 11.2 & 168 & 1.95 & 11.42 \\
\hline $10.96557+0.00971$ & 46.57 & 207.93 & 0.37 & 14.7 & 10.2 & 159 & 1.95 & 11.42 \\
\hline $10.96678+0.45096$ & 8.77 & 10.53 & 0.20 & 6.6 & 6.1 & 37 & $\ldots$ & $\ldots$ \\
\hline $10.97994+0.47065$ & 1.66 & 2.76 & 0.21 & 7.8 & 7.1 & 44 & $\ldots$ & $\ldots$ \\
\hline $10.98345+0.46966$ & 1.26 & 2.33 & 0.21 & 9.4 & 6.6 & 67 & $\ldots$ & $\ldots$ \\
\hline $10.98584+0.70115$ & 47.01 & 49.54 & 0.21 & 6.4 & 5.5 & 29 & $\ldots$ & $\ldots$ \\
\hline $10.99897+0.16847$ & 22.35 & 26.37 & 0.22 & 6.5 & 6.1 & 27 & $\ldots$ & $\ldots$ \\
\hline $11.00796+0.58933$ & 17.50 & 18.61 & 0.17 & 6.5 & 5.5 & 31 & $\ldots$ & $\ldots$ \\
\hline $11.02311+0.32766$ & 2.35 & 2.94 & 0.23 & 6.8 & 6.2 & 171 & $\ldots$ & $\ldots$ \\
\hline $11.02444+0.25564$ & 3.39 & 4.10 & 0.22 & 6.7 & 6.1 & 1 & $\ldots$ & $\ldots$ \\
\hline $11.03023+0.05336$ & 5.64 & 14.01 & 0.32 & 9.8 & 8.5 & 92 & $\ldots$ & $\ldots$ \\
\hline $11.03291+0.02761$ & 2.95 & 3.71 & 0.28 & 7.0 & 6.0 & 72 & 0.44 & 2.52 \\
\hline $11.03867-0.03903$ & 6.80 & 7.98 & 0.27 & 6.6 & 6.0 & 29 & $\ldots$ & $\ldots$ \\
\hline $11.04064+0.61702$ & 1.43 & 1.29 & 0.16 & 6.8 & 4.4 & 14 & $\ldots$ & $\ldots$ \\
\hline $11.04432+0.36909$ & 3.86 & 4.48 & 0.21 & 6.7 & 5.8 & 26 & $\ldots$ & $\ldots$ \\
\hline $11.05374+0.47840$ & 3.00 & 2.94 & 0.21 & 6.7 & 4.9 & 24 & $\ldots$ & $\ldots$ \\
\hline $11.05432+0.48628$ & 74.63 & 79.12 & 0.20 & 6.3 & 5.6 & 30 & $\ldots$ & $\ldots$ \\
\hline $11.06609+0.12835$ & 1.92 & 2.97 & 0.24 & 9.3 & 5.6 & 29 & $\ldots$ & $\ldots$ \\
\hline $11.07328+0.60324$ & 1.47 & 1.83 & 0.15 & 7.4 & 5.6 & 51 & $\ldots$ & $\ldots$ \\
\hline $11.07971+0.12145$ & 1.78 & 3.67 & 0.24 & 10.6 & 6.5 & 32 & $\ldots$ & $\ldots$ \\
\hline $11.11013-0.39894$ & 87.96 & 253.15 & 0.42 & 10.8 & 8.9 & 22 & 5.05 & 68.05 \\
\hline $11.11020-0.40122$ & 32.79 & 127.79 & 0.42 & 14.9 & 8.8 & 177 & 5.05 & 68.05 \\
\hline $11.11674-0.65359$ & 1.79 & 3.81 & 0.26 & 10.2 & 7.0 & 133 & $\ldots$ & $\ldots$ \\
\hline $11.11810-0.65584$ & 1.45 & 3.08 & 0.26 & 12.8 & 5.5 & 35 & $\ldots$ & $\ldots$ \\
\hline $11.13753-0.21337$ & 4.82 & 6.26 & 0.19 & 7.2 & 6.0 & 42 & $\ldots$ & $\ldots$ \\
\hline $11.14507-0.14098$ & 1.12 & 2.17 & 0.19 & 10.5 & 6.2 & 43 & $\ldots$ & $\ldots$ \\
\hline $11.14767+0.70009$ & 1.65 & 2.82 & 0.19 & 9.0 & 6.3 & 177 & $\ldots$ & $\ldots$ \\
\hline $11.16342+0.77554$ & 1.67 & 5.11 & 0.26 & 11.4 & 8.9 & 156 & $\ldots$ & $\ldots$ \\
\hline
\end{tabular}

NOTE. - Table 2 is published in its entirety in the electronic edition of the Astronomical Journal and is also available on the MAGPIS website (http://third.ucllnl.org/gps). A portion is shown here for guidance regarding its form and content.

a Peak flux density at $20 \mathrm{~cm}$.

b Integrated flux density at $20 \mathrm{~cm}$. An appended ' $\mathrm{E}$ ' indicates that the Gaussian model is inadequate and that the value is probably inaccurate.

c Full-width at half-maximum for major and minor axes of the elliptical Gaussian fit. The CLEAN beam $\left(6\right.$.' $^{\prime} 2 \times 5$.' $\left.^{\prime} 4\right)$ determines the size for unresolved sources.

d The $8 \mu \mathrm{m}$ and $21 \mu \mathrm{m}$ infrared fluxes come from the MSX6C catalog (Egan et al. 2003). 
TABLE 3

CATAlog of Diffuse Emission Regions

\begin{tabular}{|c|c|c|c|c|c|c|c|}
\hline $\begin{array}{c}\text { Name }(l+b) \\
\text { (1) }\end{array}$ & $\begin{array}{l}\text { Box Size } \\
\text { arcmin } \\
\text { (2) }\end{array}$ & $\begin{array}{c}\text { Scale } \\
\text { mJy/beam } \\
\text { (3) }\end{array}$ & $\begin{array}{c}S_{p} \\
\text { mJy/beam } \\
\text { (4) }\end{array}$ & $\begin{array}{c}l_{p} \\
\circ \\
(5)\end{array}$ & $\begin{array}{c}b_{p} \\
0 \\
(6)\end{array}$ & $\begin{array}{l}S_{i} \\
\text { Jy } \\
\text { (7) }\end{array}$ & $\begin{array}{c}\text { Comment } \\
\text { (8) }\end{array}$ \\
\hline $5.1917-0.2833$ & 2 & 15 & 17.0 & 5.1903 & -0.2838 & 1.05 & \\
\hline $5.4444+0.2056$ & 1 & 3 & 2.3 & 5.4466 & +0.2052 & 0.136 & \\
\hline $5.4667+0.3833$ & 6 & 1 & 1.7 & 5.5155 & +0.3474 & 1.92 & \\
\hline $5.4750-0.2458$ & 6 & 7 & 114.3 & 5.4749 & -0.2432 & 4.04 & \\
\hline $5.5250+0.0292$ & 4 & 5 & 7.5 & 5.5228 & +0.0285 & 1.04 & \\
\hline $5.9000-0.4333$ & 20 & 20 & 199.5 & 5.8851 & -0.3924 & 56.9 & \\
\hline $5.9306-0.0972$ & 2 & 4 & 3.8 & 5.9301 & -0.0992 & 0.481 & \\
\hline $5.9500+0.0833$ & 25 & 10 & 175.7 & 5.9440 & +0.2013 & 47.9 & \\
\hline $6.0158-0.3650$ & 2 & 5 & 5.5 & 6.0146 & -0.3647 & 0.765 & \\
\hline $6.0806-0.1167$ & 5 & 10 & 16.1 & 6.0818 & -0.1181 & 6.07 & \\
\hline $6.1000+0.5000$ & 25 & 4 & 7.1 & 6.1624 & +0.4969 & 17.3 & \\
\hline $6.1075-0.1731$ & 1 & 5 & 6.1 & 6.1085 & -0.1720 & 0.289 & \\
\hline $6.1500-0.6250$ & 6 & 5 & 5.1 & 6.1346 & -0.6352 & 5.25 & \\
\hline $6.2000+0.1833$ & 11 & 3 & 3.7 & 6.2235 & +0.1897 & 15.2 & \\
\hline $6.2417-0.5653$ & 4 & 5 & 82.8 & 6.2085 & -0.5475 & 1.91 & \\
\hline $6.2653-0.6458$ & 2 & 2 & 2.6 & 6.2629 & -0.6475 & 0.516 & \\
\hline $6.3333+0.5500$ & 11 & 1 & 1.6 & 6.3191 & +0.6019 & 1.99 & \\
\hline $6.4167-0.1667$ & 48 & 15 & 200.0 & 6.5753 & +0.1897 & 341 & SNR G6.4-0.1 (W28) \\
\hline $6.5000-0.4800$ & 24 & 6 & 17.2 & 6.5664 & -0.3025 & 123 & \\
\hline $6.5528-0.0958$ & 3 & 90 & 123.2 & 6.5519 & -0.0975 & 6.16 & \\
\hline $7.0167-0.2500$ & 12 & 10 & 10.4 & 6.9814 & -0.2864 & 26.7 & \\
\hline $7.0417+0.1750$ & 2 & 8 & 8.5 & 7.0419 & +0.1786 & 0.58 & \\
\hline $7.0700-0.1000$ & 17 & 5 & 3.4 & 7.2042 & -0.2347 & 18.2 & SNR G7.0-0.1 \\
\hline $7.1764+0.0875$ & 3 & 5 & 4.8 & 7.1769 & +0.0853 & 0.704 & \\
\hline $7.2167+0.1833$ & 10 & 5 & 17.2 & 7.2658 & +0.1831 & 9.10 & \\
\hline $7.2600-0.1400$ & 11 & 6 & 6.1 & 7.2536 & -0.0753 & 13.2 & \\
\hline $7.4000+0.6750$ & 10 & 3 & 3.1 & 7.3658 & +0.6853 & 4.97 & \\
\hline $7.4300+0.3500$ & 15 & 1.5 & 16.2 & 7.4203 & +0.3664 & 7.50 & \\
\hline $7.4722+0.0583$ & 1 & 150 & 218.3 & 7.4714 & +0.0581 & 2.10 & \\
\hline $7.7972-0.6333$ & 4 & 2 & 2.4 & 7.7942 & -0.6292 & 1.00 & \\
\hline $8.0750-0.1083$ & 13 & 4 & 34.0 & 8.1392 & -0.0270 & 15.2 & \\
\hline $8.1250-0.4722$ & 12 & 1.5 & 5.0 & 8.1892 & -0.3986 & 11.1 & \\
\hline $8.1417+0.2292$ & 7 & 40 & 229.5 & 8.1403 & +0.2236 & 10.7 & \\
\hline $8.2514-0.4403$ & 2 & 2 & 2.1 & 8.2498 & -0.4441 & 0.363 & \\
\hline $8.3083-0.0861$ & 6 & 15 & 263.5 & 8.3387 & -0.0931 & 7.00 & \\
\hline $8.3583-0.3000$ & 4 & 10 & 12.4 & 8.3498 & -0.3187 & 3.61 & \\
\hline $8.3750-0.3514$ & 2 & 10 & 17.0 & 8.3754 & -0.3464 & 1.28 & \\
\hline $8.4167-0.3472$ & 2 & 5 & 4.5 & 8.4170 & -0.3453 & 0.913 & \\
\hline $8.4333-0.2764$ & 2 & 7 & 7.3 & 8.4326 & -0.2781 & 0.629 & \\
\hline $8.6000-0.2500$ & 15 & 3 & 5.2 & 8.5104 & -0.2636 & 43.7 & \\
\hline $8.6625-0.3417$ & 3 & 30 & 192.3 & 8.6688 & -0.3559 & 1.60 & \\
\hline $8.7000-0.1000$ & 30 & 7 & 21.0 & 8.5265 & +0.0891 & 124 & SNR G8.7-0.1 (W30) \\
\hline $8.8583-0.2583$ & 6 & 3 & 3.4 & 8.8427 & -0.2481 & 4.79 & \\
\hline $8.8667-0.3250$ & 3 & 10 & 12.7 & 8.8722 & -0.3215 & 1.85 & \\
\hline $9.1750+0.0333$ & 6 & 3 & 8.2 & 9.1576 & +0.0162 & 3.16 & \\
\hline $9.6167+0.2000$ & 3 & 30 & 98.5 & 9.6167 & +0.1952 & 1.73 & \\
\hline $9.6319+0.4833$ & 6 & 2 & 1.6 & 9.6167 & +0.4674 & 2.00 & \\
\hline $9.6833-0.0667$ & 12 & 4 & 7.2 & 9.6639 & -0.0504 & 12.5 & \\
\hline $9.7833+0.5667$ & 16 & 3 & 5.1 & 9.7567 & +0.5380 & 8.22 & SNR G9.8+0.6 \\
\hline $9.8750-0.7500$ & 2 & 50 & 55.2 & 9.8756 & -0.7491 & 0.633 & \\
\hline $9.9667-0.7472$ & 10 & 4 & 37.5 & 10.0501 & -0.6858 & 5.99 & \\
\hline $10.0333-0.2000$ & 7 & 3 & 6.1 & 10.0629 & -0.1675 & 7.84 & \\
\hline $10.0750-0.4167$ & 4 & 10 & 9.7 & 10.0701 & -0.4180 & 3.53 & \\
\hline $10.1750-0.3667$ & 10 & 40 & 220.6 & 10.1502 & -0.3447 & 79.6 & \\
\hline $10.1833-0.0139$ & 9 & 5 & 4.4 & 10.1835 & -0.0125 & 8.70 & \\
\hline $10.2306-0.3042$ & 3 & 15 & 12.1 & 10.2291 & -0.2959 & 1.97 & \\
\hline $10.2375-0.0792$ & 4 & 5 & 4.7 & 10.2463 & -0.0770 & 2.38 & \\
\hline $10.2611+0.0750$ & 2 & 5 & 5.1 & 10.2630 & +0.0758 & 0.721 & \\
\hline $10.3139-0.1417$ & 4 & 80 & 220.5 & 10.3008 & -0.1475 & 17.8 & \\
\hline $10.3208-0.2611$ & 1 & 25 & 29.0 & 10.3213 & -0.2586 & 0.474 & \\
\hline $10.4500+0.0167$ & 4 & 20 & 114.0 & 10.4619 & +0.0341 & 3.57 & \\
\hline $10.5861-0.0417$ & 6 & 2 & 2.4 & 10.5864 & -0.0243 & 3.22 & \\
\hline $10.6333-0.4000$ & 10 & 15 & 261.4 & 10.6236 & -0.3840 & 14.5 & \\
\hline $10.6917+0.0333$ & 5 & 3 & 5.5 & 10.7026 & +0.0257 & 2.32 & \\
\hline $10.8556-0.1250$ & 3 & 3 & 2.3 & 10.8549 & -0.1243 & 0.56 & \\
\hline $10.8750+0.0875$ & 6 & 4 & 4.9 & 10.8950 & +0.0640 & 4.05 & \\
\hline $10.9639+0.0167$ & 2 & 12 & 55.7 & 10.9650 & +0.0096 & 1.10 & \\
\hline $11.0000-0.0528$ & 8 & 5 & 6.7 & 11.0384 & -0.0393 & 5.53 & \\
\hline $11.0333+0.0639$ & 2 & 15 & 51.1 & 11.0339 & +0.0629 & 0.595 & \\
\hline $11.0736-0.2264$ & 1 & 5 & 2.8 & 11.0756 & -0.2282 & 0.135 & \\
\hline $11.1639-0.7167$ & 10 & 2 & 1.9 & 11.1166 & -0.6535 & 3.11 & \\
\hline $11.1833-0.3500$ & 6 & 35 & 51.2 & 11.1778 & -0.3752 & 23.1 & SNR G11.2-0.3 \\
\hline $11.2000+0.1167$ & 10 & 5 & 8.3 & 11.2468 & +0.0691 & 10.8 & \\
\hline $11.3889-0.0667$ & 9 & 10 & 75.4 & 11.3440 & -0.0381 & 13.6 & SNR G11.4-0.1 \\
\hline
\end{tabular}


Helfand et al.

TABLE 3 - Continued

\begin{tabular}{|c|c|c|c|c|c|c|c|}
\hline $\begin{array}{c}\text { Name }(l+b) \\
\circ \\
(1)\end{array}$ & $\begin{array}{l}\text { Box Size } \\
\text { arcmin } \\
\text { (2) }\end{array}$ & $\begin{array}{c}\text { Scale } \\
\text { mJy/beam } \\
\text { (3) }\end{array}$ & $\begin{array}{c}S_{p} \\
\text { mJy/beam } \\
(4)\end{array}$ & $\begin{array}{c}l_{p} \\
\circ \\
(5)\end{array}$ & $\begin{array}{c}b_{p} \\
\circ \\
(6)\end{array}$ & $\begin{array}{l}S_{i} \\
\text { Jy } \\
\text { (7) }\end{array}$ & $\begin{array}{c}\text { Comment } \\
\text { (8) }\end{array}$ \\
\hline $11.5500+0.3333$ & 10 & 2 & 2.5 & 11.5174 & +0.3541 & 4.34 & \\
\hline $11.7708-0.0375$ & 1 & 9 & 9.7 & 11.7730 & -0.0409 & 0.247 & \\
\hline $11.8000-0.1083$ & 8 & 2 & 63.9 & 11.8369 & -0.1736 & 5.16 & \\
\hline $11.8903-0.2250$ & 6 & 2 & 4.2 & 11.8753 & -0.2370 & 3.03 & \\
\hline $11.8917+0.7500$ & 5 & 10 & 11.8 & 11.9113 & +0.7464 & 4.20 & \\
\hline $11.9500-0.0889$ & 10 & 10 & 190.0 & 11.9447 & -0.0364 & 12.6 & SNR G12.0-0.1 \\
\hline $11.9694+0.1917$ & 2 & 30 & 51.6 & 11.9686 & +0.1814 & 1.27 & \\
\hline $11.9903-0.2458$ & 6 & 5 & 4.6 & 12.0003 & -0.2542 & 2.99 & \\
\hline $12.0639-0.2819$ & 1 & 3 & 3.1 & 12.0647 & -0.2842 & 0.10 & \\
\hline $12.2083-0.1167$ & 4 & 15 & 88.3 & 12.2081 & -0.1020 & 5.20 & \\
\hline $12.2694+0.2972$ & 6 & 3 & 2.8 & 12.2759 & +0.2642 & 2.62 & \\
\hline $12.3167+0.4250$ & 4 & 2 & 3.2 & 12.3164 & +0.4292 & 0.969 & \\
\hline $12.4167+0.4667$ & 7 & 2 & 5.4 & 12.4181 & +0.5036 & 2.28 & \\
\hline $12.4361-0.0417$ & 4 & 4 & 37.2 & 12.4298 & -0.0481 & 2.52 & \\
\hline $12.5125-0.1056$ & 4 & 2 & 2.7 & 12.5070 & -0.0969 & 1.71 & \\
\hline $12.7167+0.0000$ & 8 & 6 & 7.0 & 12.6814 & +0.0058 & 8.56 & \\
\hline $12.7500+0.3333$ & 4 & 2 & 6.9 & 12.7759 & +0.3336 & 1.51 & \\
\hline $12.8000-0.1833$ & 16 & 25 & 423.2 & 12.8059 & -0.2003 & 68.0 & \\
\hline $12.8208-0.0208$ & 4 & 4 & 5.1 & 12.8198 & -0.0342 & 3.40 & \\
\hline $12.8222+0.5417$ & 3 & 2 & 13.3 & 12.8159 & +0.5575 & 0.331 & \\
\hline $12.9139-0.2806$ & 3 & 15 & 16.4 & 12.9142 & -0.2814 & 1.39 & \\
\hline $13.1875+0.0389$ & 4.5 & 20 & 27.0 & 13.1831 & +0.0514 & 7.58 & \\
\hline $13.2119+0.1411$ & 2 & 10 & 1.9 & 13.2209 & +0.1247 & 0.26 & \\
\hline $13.2306+0.0819$ & 2.5 & 5 & 5.4 & 13.2336 & +0.0758 & 1.25 & \\
\hline $13.4500+0.1389$ & 6 & 8 & 10.3 & 13.4559 & +0.1747 & 9.15 & SNR G13.5+0.2 \\
\hline $13.5389-0.1861$ & 8 & 3 & 24.6 & 13.5420 & -0.1820 & 6.26 & \\
\hline $13.7083-0.2417$ & 2 & 3 & 2.6 & 13.7065 & -0.2436 & 0.359 & \\
\hline $13.8042+0.1833$ & 4 & 3 & 3.4 & 13.8098 & +0.1847 & 1.84 & \\
\hline $13.8750+0.2819$ & 2.5 & 75 & 212.5 & 13.8726 & +0.2819 & 4.42 & \\
\hline $13.8875-0.4778$ & 2 & 5 & 4.6 & 13.8921 & -0.4752 & 0.32 & \\
\hline $13.8986-0.0153$ & 2 & 8 & 9.2 & 13.8970 & -0.0175 & 0.65 & \\
\hline $13.9931-0.1278$ & 4 & 10 & 11.1 & 13.9880 & -0.1332 & 4.64 & \\
\hline $14.1028+0.0917$ & 2 & 15 & 36.9 & 14.1048 & +0.0918 & 0.506 & \\
\hline $14.1111-0.1500$ & 12 & 5 & 16.9 & 14.2059 & -0.1104 & 25.2 & \\
\hline $14.2208-0.3083$ & 7 & 3 & 9.8 & 14.2665 & -0.2718 & 9.57 & \\
\hline $14.2300-0.5000$ & 28 & 3 & 53.1 & 14.3832 & -0.5146 & 73.0 & \\
\hline $14.3250+0.1333$ & 4 & 5 & 3.6 & 14.3316 & +0.1329 & 1.91 & \\
\hline $14.3625-0.1694$ & 7 & 4 & 3.5 & 14.3494 & -0.1510 & 8.57 & \\
\hline $14.4292-0.0639$ & 4 & 5 & 5.0 & 14.4144 & -0.0726 & 3.43 & \\
\hline $14.6167+0.0667$ & 14 & 8 & 9.7 & 14.5989 & +0.0202 & 37.6 & \\
\hline $15.0667-0.6833$ & 18 & 400 & 418.7 & 15.0340 & -0.6786 & 738 & \\
\hline $15.0764-0.1222$ & 2 & 25 & 13.0 & 15.0763 & -0.1209 & 0.436 & \\
\hline $15.4350+0.1600$ & 15 & 3 & 5.8 & 15.5197 & +0.1903 & 8.81 & \\
\hline $15.8917+0.1917$ & 7.5 & 15 & 14.3 & 15.9135 & +0.1842 & 6.54 & SNR G15.9+0.2 \\
\hline $15.9583+0.7167$ & 9 & 2 & 1.7 & 15.9146 & +0.7447 & 0.42 & \\
\hline $16.2458+0.0389$ & 2 & 2 & 1.7 & 16.2436 & +0.0419 & 0.231 & \\
\hline $16.2917-0.1667$ & 6 & 5 & 6.2 & 16.3019 & -0.1497 & 4.06 & \\
\hline $16.3583-0.1833$ & 4 & 4 & 7.9 & 16.3603 & -0.2081 & 2.48 & \\
\hline $16.4306-0.2000$ & 3 & 10 & 9.9 & 16.4442 & -0.1953 & 1.88 & \\
\hline $16.6500-0.3333$ & 12 & 4 & 11.8 & 16.6002 & -0.2758 & 12.6 & \\
\hline $16.7417+0.0833$ & 6 & 8 & 10.3 & 16.7431 & +0.0892 & 5.12 & SNR G16.7+0.1 \\
\hline $16.9417+0.7333$ & 15 & 20 & 26.2 & 16.9508 & +0.7825 & 47.9 & \\
\hline $17.0167-0.0333$ & 8 & 4 & 4.5 & 16.9603 & -0.0869 & 2.63 & \\
\hline $17.0917-0.1111$ & 6 & 3 & 13.4 & 17.1142 & -0.1125 & 2.81 & \\
\hline $17.2903-0.2022$ & 1 & 12 & 10.0 & 17.2875 & -0.1997 & 0.24 & \\
\hline $17.3361-0.1389$ & 3 & 3 & 2.9 & 17.3358 & -0.1397 & 0.282 & \\
\hline $17.3625-0.0375$ & 1.5 & 7 & 7.7 & 17.3636 & -0.0369 & 0.30 & \\
\hline $17.4458-0.0750$ & 3 & 3 & 2.4 & 17.4486 & -0.0625 & 0.717 & \\
\hline $17.5167-0.1125$ & 9 & 2 & 3.8 & 17.5614 & -0.1069 & 2.61 & \\
\hline $17.5861+0.0917$ & 2 & 2 & 2.1 & 17.5847 & +0.0919 & 0.29 & \\
\hline $17.6250+0.0472$ & 1 & 2 & 1.8 & 17.6270 & +0.0458 & 0.069 & \\
\hline $17.9278-0.6778$ & 2 & 3 & 2.5 & 17.9298 & -0.6758 & 0.185 & \\
\hline $18.0778+0.0708$ & 2 & 6 & 6.2 & 18.0792 & +0.0708 & 0.474 & \\
\hline $18.0972-0.3208$ & 1.5 & 4 & 4.0 & 18.0931 & -0.3186 & 0.19 & \\
\hline $18.1408-0.2806$ & 4 & 50 & 83.0 & 18.1465 & -0.2831 & 8.89 & \\
\hline $18.1500-0.1722$ & 9 & 8 & 7.8 & 18.1915 & -0.1725 & 9.69 & \\
\hline $18.1500+0.1000$ & 3 & 1 & 2.0 & 18.1615 & +0.0897 & 0.563 & \\
\hline $18.1889-0.3972$ & 6 & 5 & 6.4 & 18.1853 & -0.3703 & 7.27 & \\
\hline $18.2333-0.2361$ & 6 & 8 & 10.1 & 18.2610 & -0.2853 & 7.57 & \\
\hline $18.2536-0.3083$ & 5 & 12 & 15.8 & 18.2748 & -0.2903 & 8.51 & \\
\hline $18.3028-0.3894$ & 1 & 175 & 174.8 & 18.3031 & -0.3892 & 1.71 & \\
\hline $18.3292+0.0167$ & 5 & 1 & 2.1 & 18.3176 & +0.0286 & 1.72 & \\
\hline $18.3861-0.3861$ & 3 & 1.5 & 2.0 & 18.3887 & -0.3742 & 0.925 & \\
\hline $18.4417+0.0131$ & 1 & 7 & 6.2 & 18.4410 & +0.0136 & 0.114 & \\
\hline $18.4531-0.0081$ & 2 & 15 & 87.4 & 18.4615 & -0.0036 & 0.71 & \\
\hline
\end{tabular}


TABLE $3-$ Continued

\begin{tabular}{|c|c|c|c|c|c|c|c|}
\hline $\begin{array}{c}\text { Name }(l+b) \\
\circ \\
(1)\end{array}$ & $\begin{array}{l}\text { Box Size } \\
\text { arcmin } \\
(2)\end{array}$ & $\begin{array}{c}\text { Scale } \\
\text { mJy/beam } \\
\text { (3) }\end{array}$ & $\begin{array}{c}S_{p} \\
\mathrm{mJy} / \text { beam } \\
\text { (4) }\end{array}$ & $\begin{array}{c}l_{p} \\
\text { ( } \\
(5)\end{array}$ & $\begin{array}{c}b_{p} \\
\circ \\
(6)\end{array}$ & $\begin{array}{l}S_{i} \\
\text { Jy } \\
(7)\end{array}$ & $\begin{array}{c}\text { Comment } \\
\text { (8) }\end{array}$ \\
\hline $18.5181+0.3000$ & 14 & 3 & 97.1 & 18.4944 & +0.2731 & 6.96 & \\
\hline $18.6375-0.2917$ & 6 & 4 & 4.1 & 18.6077 & -0.3153 & 4.31 & \\
\hline $18.6556-0.0569$ & 1.5 & 8 & 9.7 & 18.6561 & -0.0581 & 0.275 & \\
\hline $18.6778-0.2347$ & 1.5 & 15 & 25.8 & 18.6733 & -0.2364 & 0.625 & \\
\hline $18.7361-0.1903$ & 1 & 3 & 3.0 & 18.7356 & -0.1886 & 0.173 & \\
\hline $18.7583-0.0736$ & 3 & 9 & 10.2 & 18.7600 & -0.0708 & 1.34 & \\
\hline $18.7639-0.1667$ & 3 & 3 & 3.4 & 18.7600 & -0.1636 & 1.26 & \\
\hline $18.7736+0.4000$ & 18 & 8 & 22.0 & 18.7611 & +0.2631 & 41.4 & SNR G18.8+0.3 (Kes 67) \\
\hline $18.8667-0.2667$ & 34 & 15 & 130.8 & 18.6970 & -0.4008 & 158 & \\
\hline $19.0500-0.5861$ & 5 & 7 & 12.0 & 19.0919 & -0.5874 & 2.86 & \\
\hline $19.2742+0.5444$ & 1 & 1.5 & 1.2 & 19.2747 & +0.5442 & 0.054 & \\
\hline $19.4611+0.1444$ & 9 & 7 & 105.9 & 19.4917 & +0.1353 & 8.09 & \\
\hline $19.5917+0.0250$ & 1.5 & 3 & 2.9 & 19.5923 & +0.0292 & 0.245 & \\
\hline $19.6000-0.2000$ & 15 & 7 & 306.6 & 19.6107 & -0.2342 & 24.7 & \\
\hline $19.6639-0.3042$ & 2 & 3 & 2.5 & 19.6663 & -0.2992 & 0.221 & \\
\hline $19.7139-0.2583$ & 1.5 & 3 & 2.1 & 19.7130 & -0.2564 & 0.224 & \\
\hline $19.7417+0.2800$ & 1 & 25 & 31.2 & 19.7402 & +0.2814 & 0.298 & \\
\hline $19.7600-0.5500$ & 12 & 2 & 10.3 & 19.7522 & -0.5291 & 7.46 & \\
\hline $19.7833+0.2833$ & 3 & 3 & 2.9 & 19.7836 & +0.2869 & 0.694 & \\
\hline $19.8111+0.0139$ & 4 & 1.5 & 1.7 & 19.8025 & +0.0264 & 1.06 & \\
\hline $19.9375+0.5194$ & 1 & 5 & 7.1 & 19.9364 & +0.5208 & 0.029 & \\
\hline $19.9653+0.0800$ & 1 & 2 & 2.3 & 19.9676 & +0.0858 & 0.045 & \\
\hline $19.9833-0.1833$ & 13 & 6 & 9.8 & 19.9537 & -0.2497 & 19.0 & SNR G20.0-0.2 \\
\hline $20.0764-0.1389$ & 1 & 75 & 98.4 & 20.0721 & -0.1419 & 0.863 & \\
\hline $20.0975-0.1214$ & 1 & 10 & 8.7 & 20.0994 & -0.1231 & 0.12 & \\
\hline $20.1806+0.0778$ & 15 & 1.5 & 2.8 & 20.1299 & +0.1986 & 7.57 & \\
\hline $20.2222+0.1106$ & 2 & 2 & 2.1 & 20.2217 & +0.1114 & 0.252 & \\
\hline $20.3561-0.0444$ & 1 & 2 & 1.3 & 20.3567 & -0.0447 & 0.047 & \\
\hline $20.3853-0.0208$ & 1.5 & 2 & 1.8 & 20.3828 & -0.0197 & 0.128 & \\
\hline $20.4583+0.0056$ & 6 & 1.5 & 3.9 & 20.4957 & +0.0431 & 2.04 & \\
\hline $20.4667+0.1500$ & 9 & 3 & 4.6 & 20.4095 & +0.0997 & 6.56 & \\
\hline $20.7333-0.1333$ & 14 & 12 & 30.9 & 20.7510 & -0.0899 & 24.1 & \\
\hline $20.9725-0.0711$ & 1 & 2 & 10.7 & 20.9639 & -0.0743 & 0.079 & \\
\hline $20.9917+0.0903$ & 3 & 20 & 27.1 & 20.9883 & +0.0896 & 1.69 & \\
\hline $21.0269-0.4719$ & 10 & 1 & 1.5 & 21.0216 & -0.4430 & 2.32 & \\
\hline $21.0417-0.2486$ & 6 & 1 & 3.4 & 21.0600 & -0.2354 & 1.51 & \\
\hline $21.1500-0.3000$ & 8 & 3 & 2.2 & 21.1328 & -0.3057 & 3.20 & \\
\hline $21.2486+0.0556$ & 2 & 2 & 1.3 & 21.2490 & +0.0558 & 0.097 & \\
\hline $21.3444-0.1375$ & 1 & 2 & 1.8 & 21.3435 & -0.1376 & 0.066 & \\
\hline $21.4619-0.5903$ & 4 & 2.5 & 3.0 & 21.4557 & -0.5886 & 0.816 & \\
\hline $21.5569-0.1028$ & 6 & 1 & 1.5 & 21.5430 & -0.1037 & 1.90 & \\
\hline $21.6417+0.0000$ & 5 & 2 & 5.4 & 21.6319 & -0.0070 & 1.54 & \\
\hline $21.6806-0.2333$ & 10 & 2 & 19.7 & 21.6036 & -0.1681 & 5.15 & \\
\hline $21.7078+0.0972$ & 6 & 2 & 17.5 & 21.7514 & +0.1308 & 1.98 & \\
\hline $21.8167-0.5000$ & 24 & 20 & 27.3 & 21.8202 & -0.4775 & 98.6 & SNR G21.8-0.6 (Kes 69) \\
\hline $21.8731+0.0064$ & 2 & 20 & 152.8 & 21.8747 & +0.0080 & 1.29 & \\
\hline $21.9300+0.1014$ & 1.5 & 2 & 2.1 & 21.9331 & +0.1003 & 0.21 & \\
\hline $21.9764+0.0458$ & 2 & 2 & 2.2 & 21.9753 & +0.0486 & 0.42 & \\
\hline $22.0528-0.0167$ & 15 & 1.5 & 6.4 & 22.0964 & +0.0080 & 13.2 & \\
\hline $22.2767-0.0944$ & 3 & 1.5 & 1.6 & 22.2575 & -0.0986 & 0.844 & \\
\hline $22.3069-0.0569$ & 2 & 2 & 2.8 & 22.3036 & -0.0620 & 0.439 & \\
\hline $22.3833+0.1000$ & 8 & 2 & 5.5 & 22.3597 & +0.0647 & 4.66 & \\
\hline $22.7333-0.2000$ & 30 & 7 & 61.1 & 22.9364 & -0.0736 & 130 & SNR G22.7-0.2 \\
\hline $22.7486-0.2458$ & 4 & 7 & 6.6 & 22.7603 & -0.2447 & 3.65 & \\
\hline $22.7583-0.4917$ & 5 & 12 & 20.8 & 22.7592 & -0.4775 & 6.01 & \\
\hline $22.9917-0.3583$ & 4.5 & 7 & 15.2 & 22.9742 & -0.3919 & 5.10 & \\
\hline $23.0100+0.2650$ & 9 & 1.5 & 5.0 & 23.0209 & +0.1903 & 5.24 & \\
\hline $23.1069+0.5500$ & 9 & 3 & 25.4 & 23.0675 & +0.5186 & 6.25 & \\
\hline $23.2417-0.3333$ & 30 & 20 & 45.5 & 23.2937 & -0.2775 & 122 & SNR G23.3-0.3 (W41) \\
\hline $23.2556+0.2917$ & 1.7 & 2 & 2.4 & 23.2559 & +0.2930 & 0.334 & \\
\hline $23.3750+0.4500$ & 2 & 3 & 3.0 & 23.3803 & +0.4508 & 0.35 & \\
\hline $23.4167-0.2000$ & 10 & 25 & 30.0 & 23.4359 & -0.2086 & 26.1 & \\
\hline $23.5500+0.2833$ & 16 & 3 & 3.2 & 23.5392 & +0.3214 & 20.3 & SNR G23.6+0.3 \\
\hline $23.5667-0.0333$ & 13 & 7 & 9.0 & 23.5426 & -0.0397 & 23.3 & \\
\hline $23.6583-0.2500$ & 2 & 6 & 5.6 & 23.6581 & -0.2520 & 0.501 & \\
\hline $23.6639+0.4806$ & 4 & 2 & 2.1 & 23.6848 & +0.4947 & 0.973 & \\
\hline $23.7000-0.2000$ & 3 & 7 & 10.7 & 23.7031 & -0.1959 & 1.36 & \\
\hline $23.7042+0.1722$ & 2 & 50 & 136.3 & 23.7109 & +0.1708 & 2.54 & \\
\hline $23.7417-0.0167$ & 2 & 3 & 5.7 & 23.7404 & -0.0159 & 0.329 & \\
\hline $23.7944+0.2333$ & 7 & 4 & 4.9 & 23.8265 & +0.2186 & 3.75 & \\
\hline $23.8167+0.3917$ & 3 & 2 & 2.1 & 23.8121 & +0.3814 & 0.779 & \\
\hline $23.8361+0.1042$ & 2 & 9 & 8.3 & 23.8354 & +0.1036 & 0.571 & \\
\hline $23.8667-0.1167$ & 3 & 20 & 51.2 & 23.8704 & -0.1225 & 2.31 & \\
\hline $23.9000+0.0667$ & 2 & 9 & 56.4 & 23.8982 & +0.0647 & 0.745 & \\
\hline $23.9569+0.1528$ & 3 & 15 & 206.7 & 23.9565 & +0.1497 & 3.05 & \\
\hline
\end{tabular}


Helfand et al.

TABLE 3 - Continued

\begin{tabular}{|c|c|c|c|c|c|c|c|}
\hline $\begin{array}{c}\text { Name }(l+b) \\
\circ \\
(1)\end{array}$ & $\begin{array}{l}\text { Box Size } \\
\text { arcmin } \\
\text { (2) }\end{array}$ & $\begin{array}{c}\text { Scale } \\
\text { mJy/beam } \\
\text { (3) }\end{array}$ & $\begin{array}{c}S_{p} \\
\text { mJy/beam } \\
(4)\end{array}$ & $\begin{array}{c}l_{p} \\
\circ \\
(5)\end{array}$ & $\begin{array}{c}b_{p} \\
\circ \\
(6)\end{array}$ & $\begin{array}{l}S_{i} \\
\text { Jy } \\
(7)\end{array}$ & $\begin{array}{c}\text { Comment } \\
\text { (8) }\end{array}$ \\
\hline $23.9875-0.1000$ & 2 & 6 & 20.8 & 23.9875 & -0.0893 & 0.756 & \\
\hline $24.0222+0.1917$ & 1 & 2 & 2.1 & 24.0225 & +0.1934 & 0.106 & \\
\hline $24.1200-0.0694$ & 3 & 3 & 3.3 & 24.1320 & -0.0754 & 1.58 & \\
\hline $24.1300+0.4500$ & 6 & 2 & 2.5 & 24.1325 & +0.4518 & 1.75 & \\
\hline $24.1417+0.1236$ & 3 & 9 & 72.0 & 24.1198 & +0.1262 & 1.30 & \\
\hline $24.1803+0.2167$ & 7 & 3 & 44.5 & 24.1470 & +0.1746 & 5.39 & \\
\hline $24.1969+0.2444$ & 1 & 7 & 7.4 & 24.1981 & +0.2424 & 0.243 & \\
\hline $24.3000-0.1350$ & 10 & 5 & 22.0 & 24.3366 & -0.1576 & 17.8 & \\
\hline $24.3806+0.0750$ & 7 & 7 & 12.3 & 24.4027 & +0.0685 & 10.9 & \\
\hline $24.3958+0.5778$ & 5 & 2 & 2.9 & 24.3637 & +0.5746 & 2.38 & \\
\hline $24.4750+0.4917$ & 5 & 25 & 98.2 & 24.4721 & +0.4880 & 8.37 & \\
\hline $24.5000-0.0417$ & 5 & 5 & 86.4 & 24.4922 & -0.0387 & 5.62 & \\
\hline $24.5083-0.2333$ & 9 & 7 & 105.8 & 24.5072 & -0.2221 & 17.1 & \\
\hline $24.5083+0.2333$ & 12 & 10 & 92.2 & 24.4638 & +0.2463 & 29.9 & \\
\hline $24.5583-0.1333$ & 2 & 4 & 4.4 & 24.5417 & -0.1376 & 0.888 & \\
\hline $24.6667+0.6000$ & 18 & 4 & 235.5 & 24.5410 & +0.5996 & 38.7 & SNR G24.7+0.6 \\
\hline $24.6819-0.1625$ & 3 & 25 & 46.8 & 24.6756 & -0.1537 & 4.77 & \\
\hline $24.7083-0.6333$ & 18 & 8 & 16.3 & 24.7533 & -0.7241 & 21.7 & SNR G24.7-0.6 \\
\hline $24.7167-0.0861$ & 7 & 8 & 24.3 & 24.7106 & -0.1265 & 12.8 & \\
\hline $24.7319+0.1569$ & 2 & 5 & 3.6 & 24.7356 & +0.1524 & 0.763 & \\
\hline $24.7383+0.0778$ & 3 & 6 & 7.0 & 24.7534 & +0.0624 & 2.39 & \\
\hline $24.7417-0.2083$ & 3 & 8 & 11.8 & 24.7462 & -0.2037 & 2.42 & \\
\hline $24.7931+0.0972$ & 1.5 & 60 & 83.6 & 24.7984 & +0.0963 & 2.54 & \\
\hline $24.8250+0.0917$ & 7 & 15 & 40.8 & 24.8496 & +0.0880 & 14.7 & \\
\hline 24.8319-0.1014 & 3 & 3 & 2.6 & 24.8384 & -0.1065 & 1.10 & \\
\hline $24.9528-0.0472$ & 2 & 3 & 3.1 & 24.9574 & -0.0520 & 0.575 & \\
\hline $25.1417-0.3403$ & 3 & 2 & 6.9 & 25.1568 & -0.3275 & 0.526 & \\
\hline $25.1500+0.0764$ & 4 & 8 & 11.6 & 25.1530 & +0.0891 & 2.34 & \\
\hline $25.2222+0.2917$ & 3 & 2.5 & 3.2 & 25.2185 & +0.2875 & 1.42 & \\
\hline $25.2472-0.1417$ & 3 & 50 & 579.0 & 25.2663 & -0.1609 & 3.26 & \\
\hline $25.2667-0.3167$ & 6 & 4 & 5.8 & 25.2535 & -0.3225 & 6.19 & \\
\hline $25.2917+0.3083$ & 5 & 4 & 4.9 & 25.2880 & +0.3152 & 4.27 & \\
\hline $25.3806-0.1750$ & 7 & 40 & 331.2 & 25.3980 & -0.1414 & 24.6 & \\
\hline $25.3833-0.3500$ & 8 & 3 & 4.3 & 25.3680 & -0.3653 & 8.39 & \\
\hline $25.3986+0.0292$ & 1.5 & 25 & 179.8 & 25.3947 & +0.0330 & 1.29 & \\
\hline $25.4042-0.2542$ & 4 & 7 & 7.9 & 25.4024 & -0.2536 & 2.41 & \\
\hline $25.4597-0.2083$ & 1 & 30 & 46.8 & 25.4597 & -0.2086 & 0.362 & \\
\hline $25.4694-0.1250$ & 3 & 4 & 3.9 & 25.4713 & -0.1170 & 1.22 & \\
\hline $25.4750+0.0403$ & 3 & 2 & 2.7 & 25.4763 & +0.0458 & 1.12 & \\
\hline $25.5389+0.2236$ & 2 & 3 & 5.5 & 25.5225 & +0.2162 & 0.533 & \\
\hline $25.6167-0.4597$ & 4 & 2 & 2.9 & 25.6192 & -0.4262 & 1.17 & \\
\hline $25.6528-0.0333$ & 4 & 3 & 2.6 & 25.6554 & -0.0332 & 2.12 & \\
\hline $25.6611-0.0167$ & 1 & 3.5 & 3.7 & 25.6626 & -0.0182 & 0.197 & \\
\hline $25.7056+0.0389$ & 3.5 & 15 & 16.9 & 25.7160 & +0.0485 & 4.17 & \\
\hline $25.8700+0.1350$ & 19 & 5 & 102.8 & 25.7882 & +0.0768 & 59.6 & \\
\hline $26.1000-0.0917$ & 9 & 8 & 48.0 & 26.0906 & -0.0576 & 12.6 & \\
\hline $26.1292+0.0083$ & 7 & 5 & 17.7 & 26.0823 & -0.0348 & 8.31 & \\
\hline $26.2611+0.2819$ & 2 & 2 & 2.1 & 26.2618 & +0.2735 & 0.41 & \\
\hline $26.3181-0.0111$ & 1 & 9 & 12.1 & 26.3163 & -0.0115 & 0.249 & \\
\hline $26.3292-0.0708$ & 2 & 4 & 4.6 & 26.3296 & -0.0737 & 0.631 & \\
\hline $26.4333+0.6083$ & 5 & 3 & 4.9 & 26.4301 & +0.6452 & 1.45 & \\
\hline $26.4708+0.0208$ & 3 & 7 & 19.5 & 26.4702 & +0.0213 & 1.12 & \\
\hline $26.5333+0.1833$ & 4 & 2 & 2.3 & 26.5363 & +0.1852 & 1.97 & \\
\hline $26.5375+0.4167$ & 5 & 10 & 113.1 & 26.5446 & +0.4152 & 3.67 & \\
\hline $26.5500+0.0583$ & 5 & 2 & 25.2 & 26.5358 & +0.0252 & 2.68 & \\
\hline $26.5542-0.3083$ & 12 & 3 & 96.4 & 26.6091 & -0.2119 & 15.1 & \\
\hline $26.5931+0.0944$ & 1 & 7 & 8.0 & 26.5880 & +0.0963 & 0.322 & \\
\hline $26.5972-0.0236$ & 2 & 5 & 50.9 & 26.5974 & -0.0237 & 0.697 & \\
\hline $26.6083-0.0917$ & 8 & 2 & 8.9 & 26.6413 & -0.0387 & 9.91 & \\
\hline $26.6444+0.0194$ & 1 & 7 & 7.8 & 26.6452 & +0.0197 & 0.199 & \\
\hline $26.6667-0.2000$ & 6 & 2 & 2.6 & 26.6280 & -0.1537 & 4.79 & \\
\hline $26.7208+0.1722$ & 2 & 10 & 11.3 & 26.7197 & +0.1736 & 0.584 & \\
\hline $26.8222+0.3778$ & 3 & 2 & 3.4 & 26.8180 & +0.3891 & 0.612 & \\
\hline $26.8667-0.2750$ & 2 & 3 & 2.5 & 26.8675 & -0.2714 & 0.529 & \\
\hline $26.9458+0.2083$ & 4 & 3 & 2.0 & 26.9658 & +0.2336 & 1.03 & \\
\hline $26.9500+0.0708$ & 10 & 3 & 6.1 & 26.9697 & +0.0219 & 8.22 & \\
\hline $26.9833-0.0500$ & 6 & 5 & 6.3 & 26.9736 & -0.0709 & 5.97 & \\
\hline $27.1333+0.0333$ & 11 & 5 & 5.7 & 27.1420 & -0.0125 & 17.8 & \\
\hline $27.1750-0.0708$ & 3 & 4 & 21.5 & 27.1859 & -0.0814 & 1.70 & \\
\hline $27.1875-0.1542$ & 2 & 4 & 4.9 & 27.1897 & -0.1497 & 0.884 & \\
\hline $27.2792+0.1514$ & 3 & 12 & 177.6 & 27.2797 & +0.1447 & 2.37 & \\
\hline $27.2903-0.6486$ & 1 & 2 & 1.8 & 27.2919 & -0.6486 & 0.03 & \\
\hline $27.3167-0.1250$ & 7 & 8 & 33.9 & 27.3642 & -0.1658 & 6.36 & \\
\hline $27.3375+0.1778$ & 3 & 3 & 3.3 & 27.3331 & +0.1725 & 1.35 & \\
\hline $27.3542-0.0833$ & 6 & 3 & 10.4 & 27.3164 & -0.1331 & 3.97 & \\
\hline
\end{tabular}


TABLE 3 - Continued

\begin{tabular}{|c|c|c|c|c|c|c|c|}
\hline $\begin{array}{c}\text { Name }(l+b) \\
\text { (1) }\end{array}$ & $\begin{array}{l}\text { Box Size } \\
\text { arcmin } \\
(2)\end{array}$ & $\begin{array}{c}\text { Scale } \\
\text { mJy/beam } \\
(3)\end{array}$ & $\begin{array}{c}S_{p} \\
\text { mJy/beam } \\
(4)\end{array}$ & $\begin{array}{c}l_{p} \\
\text { ( } \\
(5)\end{array}$ & $\begin{array}{c}b_{p} \\
\circ \\
(6)\end{array}$ & $\begin{array}{l}S_{i} \\
\text { Jy } \\
(7)\end{array}$ & $\begin{array}{c}\text { Comment } \\
\text { (8) }\end{array}$ \\
\hline $27.3750+0.6542$ & 3 & 2 & 1.8 & 27.3725 & +0.6564 & 0.517 & \\
\hline $27.3917-0.0111$ & 6 & 13 & 19.8 & 27.3664 & +0.0153 & 10.1 & SNR G27.4+0.0 (4C-004.71) \\
\hline $27.5000+0.1861$ & 6 & 10 & 39.2 & 27.4942 & +0.1897 & 7.71 & \\
\hline $27.5000+0.6500$ & 10 & 2.5 & 3.0 & 27.5375 & +0.6725 & 5.74 & \\
\hline $27.6833+0.0778$ & 3 & 2 & 1.6 & 27.6820 & +0.0881 & 0.432 & \\
\hline $27.7139+0.5833$ & 34 & 2 & 94.9 & 27.7020 & +0.7047 & 54.0 & SNR G27.8+0.6 \\
\hline $27.8042-0.3000$ & 6 & 2 & 7.9 & 27.7609 & -0.3403 & 1.12 & \\
\hline $27.9319+0.2056$ & 1 & 11 & 11.9 & 27.9331 & +0.2058 & 0.154 & \\
\hline $28.0125+0.3194$ & 5 & 2 & 8.5 & 27.9781 & +0.3603 & 1.55 & \\
\hline $28.0208-0.0417$ & 7 & 4 & 8.9 & 28.0314 & -0.0725 & 6.67 & \\
\hline $28.1278+0.3403$ & 2 & 4 & 3.6 & 28.1270 & +0.3408 & 0.21 & \\
\hline $28.1500+0.1583$ & 3 & 2.5 & 2.7 & 28.1514 & +0.1631 & 0.993 & \\
\hline $28.3028-0.3889$ & 2 & 24 & 24.1 & 28.3053 & -0.3853 & 1.17 & \\
\hline 28.3194-0.0222 & 2 & 4 & 3.8 & 28.3186 & -0.0125 & 0.868 & \\
\hline $28.3750+0.2028$ & 12 & 2 & 11.4 & 28.3470 & +0.1736 & 14.9 & \\
\hline $28.4333-0.0056$ & 3.5 & 5 & 38.1 & 28.4514 & +0.0025 & 2.37 & \\
\hline $28.5167+0.1333$ & 10 & 2 & 26.6 & 28.5814 & +0.1453 & 13.0 & \\
\hline $28.5167+0.3000$ & 3 & 3 & 2.6 & 28.5259 & +0.2980 & 1.34 & \\
\hline $28.5583-0.0083$ & 4.5 & 10 & 17.5 & 28.5687 & +0.0203 & 5.37 & \\
\hline $28.5972-0.3639$ & 2.5 & 12 & 15.6 & 28.5948 & -0.3591 & 1.21 & \\
\hline $28.6111+0.0139$ & 1 & 20 & 115.5 & 28.6087 & +0.0186 & 0.81 & \\
\hline $28.6111-0.1167$ & 10 & 10 & 36.3 & 28.6103 & -0.1414 & 17.7 & SNR G28.6-0.1 \\
\hline $28.6333+0.1917$ & 6 & 4 & 8.4 & 28.5831 & +0.1453 & 5.16 & \\
\hline $28.6361+0.4806$ & 2 & 6 & 6.9 & 28.6354 & +0.4769 & 0.791 & \\
\hline $28.6514+0.0292$ & 4 & 8 & 124.9 & 28.6520 & +0.0275 & 4.71 & \\
\hline $28.6958+0.0458$ & 3 & 10 & 13.4 & 28.7026 & +0.0430 & 3.26 & \\
\hline $28.7292-0.2333$ & 2 & 2 & 2.1 & 28.7281 & -0.2309 & 0.374 & \\
\hline $28.7653+0.2750$ & 3 & 5 & 3.7 & 28.7687 & +0.2741 & 1.48 & \\
\hline $28.7667-0.4250$ & 13 & 2 & 5.4 & 28.7698 & -0.4264 & 10.9 & \\
\hline $28.7972+0.2444$ & 3 & 7 & 11.4 & 28.7881 & +0.2430 & 2.16 & \\
\hline $28.8000+0.1764$ & 2 & 35 & 46.3 & 28.8070 & +0.1752 & 2.87 & \\
\hline $28.8278-0.2278$ & 4 & 5 & 5.9 & 28.8370 & -0.2509 & 2.89 & \\
\hline $28.8619+0.0625$ & 2 & 3 & 3.1 & 28.8615 & +0.0652 & 0.765 & \\
\hline $28.9000+0.2417$ & 4 & 2 & 2.4 & 28.9021 & +0.2402 & 1.98 & \\
\hline $28.9833-0.6042$ & 4 & 4 & 3.5 & 28.9727 & -0.6219 & 2.13 & \\
\hline $29.0028+0.0861$ & 4 & 4 & 5.8 & 29.0032 & +0.0702 & 2.65 & \\
\hline $29.0167+0.1722$ & 3 & 3 & 5.2 & 28.9982 & +0.1897 & 1.00 & \\
\hline $29.0667-0.6750$ & 10 & 2 & 46.1 & 29.0444 & -0.5986 & 7.56 & \\
\hline $29.0778+0.4542$ & 2 & 8 & 8.1 & 29.0771 & +0.4586 & 0.657 & \\
\hline $29.1333+0.4333$ & 8 & 3 & 3.9 & 29.1271 & +0.4363 & 7.09 & \\
\hline $29.1333-0.1458$ & 1 & 4 & 3.9 & 29.1343 & -0.1487 & 0.141 & \\
\hline $29.1583-0.0500$ & 10 & 3 & 91.7 & 29.2110 & -0.0687 & 13.3 & \\
\hline $29.2444+0.4431$ & 4 & 2 & 2.4 & 29.2366 & +0.4569 & 1.28 & \\
\hline $29.3667+0.1000$ & 12 & 4 & 6.5 & 29.3711 & +0.1035 & 16.6 & \\
\hline $29.5667+0.1083$ & 7 & 2 & 3.0 & 29.6156 & +0.0757 & 4.27 & SNR G29.6+0.1 \\
\hline $29.6181-0.5972$ & 1 & 2 & 1.5 & 29.6185 & -0.5968 & 0.05 & \\
\hline $29.7083-0.2417$ & 4 & 35 & 104.4 & 29.6890 & -0.2421 & 10.7 & SNR G29.7-0.3 (Kes 75) \\
\hline $29.9333-0.0667$ & 15 & 10 & 355.6 & 29.9563 & -0.0175 & 46.8 & \\
\hline $29.9792-0.6056$ & 3 & 2 & 1.8 & 29.9757 & -0.6069 & 0.909 & \\
\hline $30.0542-0.3389$ & 2.5 & 4 & 3.7 & 30.0563 & -0.3403 & 1.12 & \\
\hline $30.2083-0.1667$ & 4 & 6 & 503.0 & 30.2335 & -0.1381 & 4.91 & \\
\hline $30.2486+0.2431$ & 1.5 & 7 & 9.9 & 30.2468 & +0.2447 & 0.347 & \\
\hline $30.2583-0.0250$ & 4 & 5 & 6.5 & 30.2496 & -0.0192 & 3.55 & \\
\hline $30.3194-0.2083$ & 3 & 3 & 3.8 & 30.3357 & -0.2064 & 1.54 & \\
\hline $30.3667-0.2583$ & 9 & 7 & 10.8 & 30.4374 & -0.2064 & 12.7 & \\
\hline $30.3750+0.0250$ & 2.5 & 5 & 5.7 & 30.3769 & +0.0219 & 1.06 & \\
\hline $30.3778+0.1097$ & 1 & 6 & 6.9 & 30.3785 & +0.1075 & 0.177 & \\
\hline $30.3819-0.1097$ & 1 & 8 & 13.7 & 30.3830 & -0.1108 & 0.315 & \\
\hline $30.4583+0.4333$ & 7 & 2 & 5.6 & 30.4707 & +0.4736 & 4.28 & \\
\hline $30.5083-0.3292$ & 7 & 5 & 5.0 & 30.5102 & -0.2997 & 8.26 & \\
\hline $30.5333+0.0208$ & 1.5 & 15 & 199.7 & 30.5346 & +0.0208 & 1.56 & \\
\hline $30.5583-0.5000$ & 12 & 4 & 47.5 & 30.5363 & -0.4436 & 17.2 & \\
\hline $30.7050-0.2750$ & 4.5 & 20 & 34.7 & 30.6874 & -0.2608 & 7.43 & \\
\hline $30.7500-0.0500$ & 13 & 50 & 267.1 & 30.7196 & -0.0825 & 142 & \\
\hline $30.7667-0.2250$ & 3 & 6 & 8.9 & 30.7691 & -0.2197 & 2.62 & \\
\hline $30.7958+0.1653$ & 1 & 10 & 12.6 & 30.7974 & +0.1686 & 0.376 & \\
\hline $30.8333-0.2069$ & 5 & 6 & 7.8 & 30.8541 & -0.1931 & 5.67 & \\
\hline $30.8486+0.1333$ & 4 & 10 & 90.4 & 30.8663 & +0.1142 & 3.81 & \\
\hline $30.8903+0.1764$ & 1 & 3 & 2.7 & 30.8908 & +0.1753 & 0.189 & \\
\hline $30.9069+0.1611$ & 1 & 4 & 4.3 & 30.9063 & +0.1625 & 0.209 & \\
\hline $30.9500+0.5417$ & 2 & 7 & 7.2 & 30.9513 & +0.5397 & 0.60 & \\
\hline $30.9569+0.0819$ & 1 & 20 & 36.2 & 30.9580 & +0.0869 & 0.526 & \\
\hline $30.9639+0.5917$ & 4 & 4 & 4.6 & 30.9519 & +0.5919 & 2.09 & \\
\hline $31.0000-0.0500$ & 6 & 4 & 4.4 & 31.0252 & -0.0653 & 7.34 & \\
\hline $31.0083+0.5500$ & 5 & 2 & 1.9 & 31.0447 & +0.5081 & 1.92 & \\
\hline
\end{tabular}


Helfand et al.

TABLE 3 - Continued

\begin{tabular}{cccrcccc}
\hline \hline $\begin{array}{c}\text { Name }(l+b) \\
0\end{array}$ & $\begin{array}{c}\text { Box Size } \\
\text { arcmin } \\
(2)\end{array}$ & $\begin{array}{c}\text { Scale } \\
\text { my/beam } \\
(3)\end{array}$ & $\begin{array}{c}S_{p} \\
\text { mJy/beam } \\
(4)\end{array}$ & $\begin{array}{c}l_{p} \\
0\end{array}$ & $\begin{array}{c}b_{p} \\
0\end{array}$ & $\begin{array}{c}S_{i} \\
\text { Jy } \\
(6)\end{array}$ & Comment \\
$(7)$ & $(8)$ \\
\hline $31.0500+0.0833$ & 3 & 10 & 17.8 & 31.0596 & +0.0919 & 3.17 & \\
$31.0583+0.4833$ & 6 & 5 & 11.6 & 31.0497 & +0.4697 & 4.86 & \\
$31.0653+0.0444$ & 2 & 10 & 83.2 & 31.0702 & +0.0508 & 1.44 & \\
$31.0708-0.0444$ & 2 & 4 & 4.9 & 31.0741 & -0.0458 & 1.21 & \\
$31.0786+0.0236$ & 1.5 & 4 & 4.1 & 31.0774 & +0.0247 & 0.636 & \\
$31.1217+0.0486$ & 3 & 4 & 6.4 & 31.1219 & +0.0631 & 2.44 & \\
$31.1319+0.2889$ & 4 & 6 & 8.3 & 31.1308 & +0.2686 & 2.90 & \\
$31.1750-0.1083$ & 7 & 4 & 15.7 & 31.1874 & -0.0647 & 7.50 & \\
$31.2486-0.0278$ & 6 & 3 & 3.8 & 31.2596 & -0.0231 & 5.81 & \\
$31.2736+0.4778$ & 3 & 2 & 1.6 & 31.2669 & +0.4886 & 0.556 & \\
$31.2972+0.0500$ & 3 & 3 & 99.1 & 31.2796 & +0.0631 & 1.76 & \\
$31.3708-0.0361$ & 3 & 3 & 2.6 & 31.3702 & -0.0464 & 1.17 & \\
$31.4000-0.2667$ & 3 & 15 & 36.0 & 31.3947 & -0.2592 & 2.31 & \\
$31.4667-0.3444$ & 3 & 9 & 9.9 & 31.4758 & -0.3453 & 1.24 & \\
$31.4903+0.3708$ & 1 & 4 & 2.6 & 31.4913 & +0.3680 & 0.12 & \\
$31.5417-0.1056$ & 3 & 2 & 2.0 & 31.5402 & -0.1070 & 0.772 & \\
$31.5500-0.7167$ & 22 & 2 & 20.5 & 31.3725 & -0.7514 & 7.65 & SNR G31.5-0.6 \\
$31.6097+0.3347$ & 4.5 & 3 & 3.7 & 31.6219 & +0.3319 & 1.74 & \\
$31.6125-0.2389$ & 4 & 2 & 2.2 & 31.6258 & -0.2436 & 1.31 & \\
$31.7278+0.7000$ & 1 & 2 & 1.8 & 31.7270 & +0.6975 & 0.057 & \\
$31.8208-0.1222$ & 3 & 3 & 3.2 & 31.8241 & -0.1136 & 0.896 & \\
$31.8667+0.0111$ & 8 & 25 & 73.2 & 31.8680 & +0.0641 & 27.6 & SNR G31.9+0.0 (3C391) \\
$31.9069-0.3083$ & 1 & 3 & 3.0 & 31.9053 & -0.3081 & 0.076 & \\
$31.9417+0.1778$ & 5 & 2 & 7.4 & 31.9097 & +0.1952 & 1.19 & \\
& & & & & & & \\
\hline
\end{tabular}


TABLE 4

High-PRobability SuPERnOVA REMnant CANDIDATES

\begin{tabular}{|c|c|c|c|c|}
\hline $\begin{array}{c}\text { Name }(l+b) \\
\text { (1) }\end{array}$ & $\begin{array}{l}\text { Diameter } \\
\text { arcmin } \\
(2)\end{array}$ & $\begin{array}{l}\text { Box Size } \\
\text { arcmin } \\
(3)\end{array}$ & $\begin{array}{c}S_{p} \\
\text { mJy/beam } \\
(4)\end{array}$ & $\begin{array}{l}S_{i} \\
\text { Jy } \\
(5)\end{array}$ \\
\hline $6.4500-0.5583$ & 3.3 & 6.0 & 4.7 & 6.64 \\
\hline $6.5375-0.6028$ & 5.0 & 9.0 & 16.9 & 9.42 \\
\hline $7.2167+0.1833$ & 6.5 & 10.0 & 17.2 & 9.10 \\
\hline $8.3083-0.0861$ & 3.0 & 6.0 & 263.5 & 7.00 \\
\hline $8.8583-0.2583$ & 4.0 & 6.0 & 3.4 & 4.79 \\
\hline $9.6833-0.0667$ & 8.5 & 12.0 & 7.2 & 12.5 \\
\hline $10.8750+0.0875$ & 2.8 & 6.0 & 4.9 & 4.05 \\
\hline $11.1639-0.7167$ & 7.0 & 10.0 & 1.9 & 3.11 \\
\hline $11.2000+0.1167$ & 7.5 & 10.0 & 8.3 & 10.8 \\
\hline $11.5500+0.3333$ & 4.5 & 10.0 & 2.5 & 4.34 \\
\hline $11.8903-0.2250$ & 3.5 & 6.0 & 4.2 & 3.03 \\
\hline $12.2694+0.2972$ & 4.0 & 6.0 & 2.8 & 2.62 \\
\hline $12.7167+0.0000$ & 4.5 & 8.0 & 7.0 & 8.56 \\
\hline $12.8208-0.0208$ & 2.0 & 4.0 & 5.1 & 3.40 \\
\hline $12.9139-0.2806$ & 1.5 & 3.0 & 16.4 & 1.39 \\
\hline $13.1875+0.0389$ & 2.5 & 4.5 & 27.0 & 7.58 \\
\hline $16.3583-0.1833$ & 2.8 & 4.0 & 7.9 & 2.48 \\
\hline $17.0167-0.0333$ & 4.0 & 8.0 & 4.5 & 2.63 \\
\hline $17.3361-0.1389$ & 1.8 & 3.0 & 2.9 & 0.282 \\
\hline $18.1500-0.1722$ & 7.0 & 9.0 & 7.8 & 9.69 \\
\hline $18.2536-0.3083$ & 3.5 & 5.0 & 15.8 & 8.51 \\
\hline $18.6375-0.2917$ & 4.0 & 6.0 & 4.1 & 4.31 \\
\hline $18.7583-0.0736$ & 1.6 & 3.0 & 10.2 & 1.34 \\
\hline $19.4611+0.1444$ & 6.0 & 9.0 & 105.9 & 8.09 \\
\hline $19.5800-0.2400$ & 3.2 & 5.0 & 306.6 & 6.61 \\
\hline $19.5917+0.0250$ & 0.8 & 1.5 & 2.9 & 0.245 \\
\hline $19.6100-0.1200$ & 4.5 & 6.0 & 8.5 & 5.87 \\
\hline $19.6600-0.2200$ & 4.5 & 6.0 & 10.2 & 4.80 \\
\hline $20.4667+0.1500$ & 5.5 & 9.0 & 4.6 & 6.56 \\
\hline $21.5569-0.1028$ & 4.0 & 6.0 & 1.5 & 1.90 \\
\hline $21.6417+0.0000$ & 2.8 & 5.0 & 5.4 & 1.54 \\
\hline $22.3833+0.1000$ & 7.0 & 8.0 & 5.5 & 4.66 \\
\hline $22.7583-0.4917$ & 3.8 & 5.0 & 20.8 & 6.01 \\
\hline $22.9917-0.3583$ & 3.8 & 4.5 & 15.2 & 5.10 \\
\hline $23.5667-0.0333$ & 9.0 & 13.0 & 9.0 & 23.3 \\
\hline $24.1803+0.2167$ & 5.2 & 7.0 & 44.5 & 5.39 \\
\hline $25.2222+0.2917$ & 2.0 & 3.0 & 3.2 & 1.42 \\
\hline $27.1333+0.0333$ & 11.0 & 11.0 & 5.7 & 17.8 \\
\hline $28.3750+0.2028$ & 10.0 & 12.0 & 11.4 & 14.9 \\
\hline $28.5167+0.1333$ & 14.0 & 10.0 & 26.6 & 13.0 \\
\hline $28.5583-0.0083$ & 3.0 & 4.5 & 17.5 & 5.37 \\
\hline $28.7667-0.4250$ & 9.5 & 13.0 & 5.4 & 10.9 \\
\hline $29.0667-0.6750$ & 8.0 & 10.0 & 46.1 & 7.56 \\
\hline $29.0778+0.4542$ & 0.7 & 2.0 & 8.1 & 0.657 \\
\hline $29.3667+0.1000$ & 9.0 & 12.0 & 6.5 & 16.6 \\
\hline $30.8486+0.1333$ & 2.2 & 4.0 & 90.4 & 3.81 \\
\hline $31.0583+0.4833$ & 4.5 & 6.0 & 11.6 & 4.86 \\
\hline $31.6097+0.3347$ & 3.1 & 4.5 & 3.7 & 1.74 \\
\hline $31.8208-0.1222$ & 1.8 & 3.0 & 3.2 & 0.896 \\
\hline
\end{tabular}

